\title{
Angular momentum projection for a Nilsson mean-field plus pairing model
}

\author{
Yin Wang, ${ }^{1,2}$ Feng Pan ${ }^{*},{ }^{1,3}$ Kristina D. Launey ${ }^{3}$ Yan-An Luo, ${ }^{4}$ and J. P. Draayer ${ }^{3}$ \\ ${ }^{1}$ Department of Physics, Liaoning Normal University, Dalian 116029, China \\ ${ }^{2}$ Department of Physics, Chifeng University, Chifeng 024000, China \\ ${ }^{3}$ Department of Physics and Astronomy, Louisiana State University, Baton Rouge, LA 70803-4001, USA \\ ${ }^{4}$ School of Physics, Nankai University, Tianjin 300071, P. R. China
}

\begin{abstract}
The angular momentum projection for the axially deformed Nilsson mean-field plus a modified standard pairing (MSP) or the nearest-level pairing (NLP) model is proposed. Both the exact projection, in which all intrinsic states are taken into consideration, and the approximate projection, in which only intrinsic states with $K=0$ are taken in the projection, are considered. The analysis shows that the approximate projection with only $K=0$ intrinsic states seems reasonable, of which the configuration subspace considered is greatly reduced. As simple examples for the model application, low-lying spectra and electromagnetic properties of ${ }^{18} \mathrm{O}$ and ${ }^{18} \mathrm{Ne}$ are described by using both the exact and approximate angular momentum projection of the MSP or the NLP, while those of ${ }^{20} \mathrm{Ne}$ and ${ }^{24} \mathrm{Mg}$ are described by using the approximate angular momentum projection of the MSP or NLP.
\end{abstract}

PACS numbers: 21.60.Fw, 21.60.Cs, 27.30.+t

\section{INTRODUCTION}

It is well known that the spherical harmonic oscillator (HO) shell model is a well-established theory to elucidate the structure of atomic nuclei, at least in low-energy regimes [1, 2]. Through the pioneering work of Bohr [3], Belyaev [4], and Elliott [5,6], it is commonly believed that the spherical shell model can be used to describe lowlying spectroscopy of nuclei, when the most important residual interactions, such as the quadrupole-quadrupole and pairing interactions, are taken into consideration. However, to date this approach has been limited to relativity light nuclei as computational resources are currently inadequate for medium mass and heavier nuclei since the size of the model space and computational requirements grow combinatorially with an increase in the number of particles and active orbitals. In order to reduce the dimension of the configuration subspace to make shell-model solutions more feasible, some innovative alternatives have been advanced. Examples include the SU(3) shell model developed mainly by Elliott for light nuclei [5, 6], the pseudo $\mathrm{SU}(3)$ model for heavy nuclei [7], and its symplectic $\mathrm{Sp}(6, \mathrm{R})$ and pseudosymplectic extensions [8-11], as well as the recently developed no-core symplectic shell model, NCSpM [12], and $a b$ initio symmetry-adapted no-core shell model, SA-NCSM [13], which utilize an $\mathrm{Sp}(6, \mathrm{R})$-based model space selection. These symmetry-adapted approaches employ only a subset of important shell-model states, within which the shellmodel structures, once calculations converge, can be well understood. On the other hand, self-consistent methods for deriving the nuclear mean-field with density dependent effective interactions [14-16] or relativistic mean-field considerations [17-19] based on the HF and HFB approximations have been well developed [20].

Since the quadrupole-quadrupole interaction is an important part of the residual shell model interaction that tracks with nuclear deformation and rotational spectra as demonstrated by the Elliott model [5, 6], Nilsson et al. [21, 22] extended the spherical shell model to the deformed case by introducing a deformation-dependent oscillator length. This provides for a simple description of deformed nuclei and their rotational structure in a (deformed) shell-model basis. Not only ground-state properties of deformed nuclei can be correctly described within the framework of the Nilsson model as shown in $[21,22]$, but also the deformed basis can be used to simplify spherical shell-model calculations through angular momentum projection [23-25] on the deformed basis through which the dimension of the shell-model subspace is greatly reduced, especially for well-deformed nuclei.

Because the deformation introduced in the Nilsson model is intrinsic, which is equivalent to a rotational symmetryviolating mean-field description, rotational symmetry restoration or angular momentum projection is required. The deformed description is reasonable since the long-range quadrupole-quadrupole interaction is taken into account [2]. It is therefore interesting to see whether low-lying spectra of deformed nuclei can indeed be described by the deformed Nilsson model after angular momentum projection. It should be emphasized that our approach is different from those shown in [23-25], in which the Hamiltonian used is rotationally invariant based on the spherical shell model, and only the product of the deformed Nilsson single-particle states, or quasi-particle states when pairing interaction is treated by the BCS approximation, are projected in the diagonalization. In the present study the Hamiltonian used

* e-mail: daipan@dlut.edu.cn 
is the intrinsic deformed Nilsson mean field plus pairing interaction, which breaks the rotational symmetry, and the angular momentum projection is carried out for the intrinsic deformed Hamiltonian, but not directly for the product of the single-particle states considered in the diagonalization.

In Sec. II, we briefly review the Nilsson mean-field plus a modified standard pairing model and the nearest-level pairing model, which are all exactly solvable. The angular momentum projection for the deformed Hamiltonian is described in Sec. III. In Sec. IV, as simple examples of the application, low-lying spectra and E2 properties of ${ }^{18} \mathrm{O}$ and ${ }^{18} \mathrm{Ne}$ are studied by using both the exact and the approximate angular momentum projection, while those of ${ }^{20} \mathrm{Ne}$ are only studied by using the approximate angular momentum projection. As a further test of the applicability of the model, some low-lying lever energies and $\mathrm{B}(\mathrm{E} 2)$ values of ${ }^{24} \mathrm{Mg}$, which is described within the $d s$-shell with 4 valence protons and 4 valence neutrons, are fitted with less model parameters. A summary is given in Sec. V.

\section{EXACTLY SOLVABLE NILSSON MEAN-FIELD PLUS PAIRING MODELS}

Within the deformed mean field, there are several types of pairing interactions that can be solved exactly. The pairing interaction that is most frequently adopted in mean-field theories is standard pairing; namely, identical pairing strength between all like-nucleon pairs. In this case, the exact solutions can be formulated by using the RichardsonGaudin methods [26, 27]. It should be stated that the model, in which pairing interactions with different 'on-site' interaction strengths are included, is also exactly solvable using the same formalism, which will be called the modified standard pairing (MSP).

The Hamiltonian of the deformed Nilsson mean-field plus the MSP model is given by

$$
\hat{H}_{\mathrm{MSP}}=\sum_{i} \varepsilon_{i} \hat{n}_{i}-\sum_{i} G_{i i} b_{i}^{\dagger} b_{i}-G \sum_{i i^{\prime}} b_{i}^{\dagger} b_{i^{\prime}},
$$

where the sums run over the levels considered of total number $N, G>0$ is the overall pairing strength, $G_{i i}+G>0$ $(i=1,2, \cdots, N)$ are the 'on-site' pairing interaction strengths, $\varepsilon_{i}$ are nondegenerate single-particle energies generated from the Nilsson shell model, of which the parameters used are shown in Appendix A, $\hat{n}_{i}=a_{i}^{\dagger} a_{i}+a_{i}^{\dagger} a_{\bar{i}}$ is the number operator for valence particles in the $i$-th Nilsson level, and $b_{i}^{\dagger}=a_{i}^{\dagger} a_{\bar{i}}^{\dagger}\left(b_{i}=a_{i} a_{i}\right)$ are pair creation (annihilation) operators, in which $a_{i}^{\dagger}$ is the $i$-th level single-particle creation operator in the Nilsson model and $a_{i}^{\dagger}$ the corresponding time-reversed state. The $b_{i}^{\dagger}$ and $b_{i}$ satisfy the following commutation relations:

$$
\left[b_{i}, b_{i^{\prime}}^{\dagger}\right]=\delta_{i i^{\prime}}\left(1-2 N_{i}\right), \quad\left[N_{i}, b_{i^{\prime}}^{\dagger}\right]=\delta_{i i^{\prime}} b_{i^{\prime}}^{\dagger}, \quad\left[N_{i}, b_{i^{\prime}}\right]=-\delta_{i i^{\prime}} b_{i^{\prime}}
$$

where $N_{i}=\hat{n}_{i} / 2$ is the pair number operator in the $i$-th level. According to the Richardson-Gaudin method, $k$-pair eigenstates of (1) can be written as

$$
\left|k ; \xi, n_{q_{1}}, n_{q_{2}}, \cdots, n_{q_{r}}\right\rangle=S^{+}\left(x_{1}^{(\xi)}\right) S^{+}\left(x_{2}^{(\xi)}\right) \cdots S^{+}\left(x_{k}^{(\xi)}\right)\left|n_{q_{1}}, n_{q_{2}}, \cdots, n_{q_{r}}\right\rangle,
$$

where $n=2 k+\sum_{\rho=1}^{r} n_{q_{\rho}}$ is the total number of valence particles, $q_{1}, q_{2}, \cdots, q_{r}$ are the levels occupied by $r$ single particles with $n_{q_{\rho}}=1(\rho=1,2, \cdots, r)$,

$$
S^{+}(x)=\sum_{i} \frac{1}{2 \varepsilon_{i}-G_{i i}-x} b_{i}^{\dagger}
$$

in which $x$ is the spectral parameter to be determined, and $\left|n_{q_{1}}, n_{q_{2}}, \cdots, n_{q_{r}}\right\rangle$ is the pairing vacuum state satisfying $b_{i}\left|n_{q_{1}}, n_{q_{2}}, \cdots, n_{q_{r}}\right\rangle=0$ for all $i$ due to the Pauli exclusion principle.

It can then be verified by using the commutation relations given by (2) that (3) is the eigenstates of (1) only when the spectral parameters $x_{s}$ satisfy the following set of equations:

$$
1-G \sum_{i} \frac{1-\sum_{\rho} n_{q_{\rho}} \delta_{i q_{\rho}}}{2 \varepsilon_{i}-G_{i i}-x_{s}}-2 G \sum_{s^{\prime} \neq s} \frac{1}{x_{s}-x_{s^{\prime}}}=0
$$

for $s=1,2, \cdots, k$. For the $\xi$-th set of solution $\left\{x_{s}^{(\xi)}\right\}$ of Eq. (5), the corresponding eigen-energy of (1) is given by

$$
E_{k}^{(\xi)}=\sum_{s=1}^{k} x_{s}^{(\xi)}+\sum_{\rho} \varepsilon_{q_{\rho}} n_{q_{\rho}}
$$


According to Heine-Stieltjes correspondence [28], for nonzero pairing strength $G$, zeros of the extended HeineStieltjes polynomials $y(x)$ are roots of Eq. (5), where $y(x)$ should satisfy the following second-order Fuchsian equation:

$$
A(x) y^{\prime \prime}(x)+B(x) y^{\prime}(x)-V(x) y(x)=0 .
$$

Here, $A(x)=\prod_{i}\left(x-2 \varepsilon_{i}+G_{i i}\right)$ is a polynomial of degree $N$, the polynomial $B(x)$ is given as

$$
B(x) / A(x)=-\sum_{i} \frac{1-\sum_{\rho} n_{q_{\rho}} \delta_{i q_{\rho}}}{x-2 \varepsilon_{i}+G_{i i}}-\frac{1}{G},
$$

where the sum runs over all Nilsson levels considered, and $V(x)$ is a Van Vleck polynomial of degree $N-1$, which is determined according to Eq. (7). Therefore, the polynomial approach for the standard pairing model proposed in $[28,29]$ applies to the modified standard pairing model as well.

As shown in [30], the standard pairing interaction [26, 28], which is usually used in applications with the BCS or the HFB approximation [31, 32], is not a particularly good approximation for well-deformed nuclei. The nearest-level pairing interaction is inspired by the level-dependent Gaussian-type pairing interaction with

$$
G_{i i^{\prime}}=-A_{i i^{\prime}} e^{-B\left(\varepsilon_{i}-\varepsilon_{i^{\prime}}\right)^{2}}
$$

introduced in [30], where $i$ and $i^{\prime}$ each represents doubly occupied Nilsson levels with single-particle energies $\varepsilon_{i}$ and $\varepsilon_{i^{\prime}}$, generated from the Nilsson model, the parameters $A_{i i^{\prime}}>0$ and $B>0$ are adjusted in such a way that the location of the first excited eigen-solution lies approximately at the same energy as for the standard pairing case. Of course, there is some freedom in adjusting the parameters, allowing one to control in a phenomenological way the interaction among the levels. The expression (9) implies that scattering between particle pairs occupying levels with single-particle energies that lie close are favored; scattering between particle pairs in levels with distant single-particle energies are unfavored. As an approximation, this pairing interaction was further simplified to the nearest-level coupling in [33-35], namely, $G_{i i^{\prime}}$ is given by

$$
G_{i i}=-A_{i i}, G_{i i+1}=G_{i+1 i}=-A e^{-B\left(\varepsilon_{i}-\varepsilon_{i+1}\right)^{2}},
$$

where $A_{i i} \geq 0$ and $A>0$ are real parameters, while $G_{i i^{\prime}}$ are taken to be 0 otherwise. Hence, the deformed mean-field plus the nearest-level pairing (NLP) Hamiltonian can be expressed as

$$
\hat{H}_{\mathrm{NLP}}=\sum_{\rho=1}^{r} \varepsilon_{q_{\rho}} \hat{n}_{q_{\rho}}+\sum_{i, i^{\prime}}^{\prime} t_{i i^{\prime}} b_{i}^{\dagger} b_{i^{\prime}},
$$

where the first sum runs over the levels occupied by a single particle which occurs in the description of odd-A nuclei or several unpaired particles in broken-pair cases, and the second primed sum runs only over levels that are occupied by pairs of particles. For the nearest-level pairing interaction, the $t$-matrix is tridiagonal given by $t_{i i}=2 \varepsilon_{i}+G_{i i}=2 \varepsilon_{i}-A_{i i}$ and $t_{i i+1}=t_{i+1 i}=G_{i i+1}$ with $t_{i i^{\prime}}=0$ otherwise.

The eigenstates of (11) for $k$-pair excitation can be expressed as

$$
\left|k ; \eta, n_{q_{1}}, n_{q_{2}}, \cdots, n_{q_{r}}\right\rangle=\sum_{i_{1}<i_{2} \cdots<i_{k}}^{\prime} C_{i_{1} i_{2} \cdots i_{k}}^{(\eta)} b_{i_{1}}^{\dagger} b_{i_{2}}^{\dagger} \cdots b_{i_{k}}^{\dagger}\left|n_{q_{1}}, n_{q_{2}}, \cdots, n_{q_{r}}\right\rangle,
$$

where $n=2 k+\sum_{\rho=1}^{r} n_{q_{\rho}}, q_{1}, q_{2}, \cdots, q_{r}$ are the levels occupied by $r$ single particles, the prime indicates that $i_{1}, i_{2}, \cdots, i_{k}$ can not be taken to be $q_{1}, q_{2}, \cdots, q_{r}$ in the summation, and $n_{q_{\rho}}=1(\rho=1,2, \cdots, r)$. If only even-even and odd-A nuclei are treated without including broken-pair cases, $r$ is taken to be 1 for odd-A nuclei, and 0 for even-even nuclei. In (12), $C_{i_{1} i_{2} \cdots i_{k}}^{(\eta)}$ is a determinant given by

$$
C_{i_{1} i_{2} \cdots i_{k}}^{(\eta)}=\left|\begin{array}{cccc}
g_{i_{i}}^{\eta_{1}} & g_{i_{2}}^{\eta_{1}} & \cdots & g_{i_{k}}^{\eta_{1}} \\
g_{i_{i}}^{\eta_{2}} & g_{i_{2}}^{\eta_{2}} & \cdots & g_{i_{k}}^{\eta_{2}} \\
\cdots & \cdots & \cdots & \cdots \\
g_{i_{i}}^{\eta_{k}} & g_{i_{2}}^{\eta_{k}} & \cdots & g_{i_{k}}^{\eta_{k}}
\end{array}\right|,
$$

where $\eta$ is a shorthand notation for a selected set of $k$ eigenvalues of the $t$ matrix without the corresponding $r$ rows and columns, denoted as $\tilde{t}$, which can be used to distinguish the eigenstates with the same number of pairs, $k$, and $g^{\eta_{p}}$ is the $p$-th eigenvector of the $\tilde{t}$ matrix. 
The excitation energies corresponding to (12) can be expressed as

$$
E_{k}^{(\eta)}=\sum_{\rho=1}^{r} \varepsilon_{q_{\rho}}+\sum_{i=1}^{k} E^{\left(\eta_{i}\right)}
$$

where the first sum runs over $r$ Nilsson levels each occupied by a single valence nucleon, which occurs in odd-A nuclei or in broken-pair cases, the second one is a sum of $k$ different eigenvalues of the $\tilde{t}$-matrix. Obviously, $\tilde{t}$ is a $(N-r) \times(N-r)$ matrix, since those levels occupied by single valence nucleons are excluded resulting from the Pauli blocking. $E^{\left(\eta_{p}\right)}$ is the $p$-th eigenvalue of the $\tilde{t}$-matrix, that is

$$
\sum_{i^{\prime}} \tilde{t}_{i i^{\prime}} g_{i^{\prime}}^{\eta_{p}}=E^{\left(\eta_{p}\right)} g_{i}^{\eta_{p}}
$$

For even-even nuclei, the $k$-pair excitation energies are determined by the sum of $k$ different eigenvalues chosen from the $N$ eigenvalues of the $\tilde{t}$ matrix with $r=0$, the total number of excited levels is $N ! / k !(N-k)$ !. While for odd-A nuclei or broken-pair cases, the Nilsson level that is occupied by the single valence nucleon should be excluded in the original $t$ matrix. In the latter case, the eigenvalue problem of (11) can be solved simply by diagonalizing the corresponding $\tilde{t}$ matrix as shown in (15) with the eigenstates and the corresponding eigen-energy given by (12) and (14), respectively.

Since the Nilsson mean-field plus the MSP or the NLP model is exactly solvable, the angular momentum projection of these two models will be considered in the following.

\section{THE ANGULAR MOMENTUM PROJECTION}

(1) An exact angular momentum projection. For a system with $n$ valence particles, according to the general projection procedure, let $\{|\tau J K\rangle\}$ be a set of normalized orthogonal intrinsic states with good angular momentum $J$ and the quantum number $K$ of its third component in the intrinsic frame, which may be expanded in terms of $n$ single-particle creation operators in the spherical shell model coupled to angular momentum $J$ or in terms of eigenstates $\{|n, \xi\rangle\}$ of the Nilsson plus pairing model Hamiltonian $\hat{H}$ given by (1) or (11), where $\xi$ stands for a set of quantum numbers needed. The deformed intrinisc Hamiltonian $\hat{H}$ can be projected into a subspace with good angular momentum. The projected Hamiltonian can be written as

$$
\tilde{H}=\sum_{J M K} \frac{1}{2 J+1} P_{M K}^{J} \hat{H} P_{K M}^{J}
$$

where $[2,25,36]$

$$
\begin{gathered}
P_{M K}^{J}=\frac{2 J+1}{8 \pi^{2}} \int d \alpha_{1} \sin \alpha_{2} d \alpha_{2} d \alpha_{3} D_{M K}^{J}\left(\alpha_{1}, \alpha_{2}, \alpha_{3}\right) e^{i \alpha_{1} \hat{J}_{z^{\prime}}} e^{i \alpha_{2} \hat{J}_{y^{\prime}}} e^{i \alpha_{3} \hat{J}_{z^{\prime}}}=\sum_{\tau}|\tau J M\rangle_{\mathrm{L}}\langle\tau J K|, \\
P_{K M}^{J}=\left(P_{M K}^{J}\right)^{\dagger}=\sum_{\tau}|\tau J K\rangle_{\mathrm{L}}\langle\tau J M|
\end{gathered}
$$

are generalized projection operators, in which $|\tau J M\rangle_{\mathrm{L}}$ are eigenstates of the total angular momentum and its third component in the laboratory frame. It should be stated that (16) is projected from the rotation symmetry breaking intrinsic deformed Nilsson mean field plus the pairing Hamiltontian, which is different from the previous angular momentum projection procedure used in [25, 36]. In [25, 36], the Hamiltonian used is rotational invariant, for which the angular momentum projection is only carried out for several intrinsic states to diagonalize the rotational invariant Hamiltonian or calculate the expectation values of excitation energies according to the variational principle. The factor $1 /(2 J+1)$ in the sum of $(16)$ ensures that $\tilde{H}=\hat{H}$ when $\hat{H}$ is rotational invariant. It is clear that, with the projection, $\tilde{H}$ given in (16) can be diagonalized within a subspace spanned by the basis vectors $\left\{|\tau J M\rangle_{\mathrm{L}}\right\}$ in the laboratory frame, of which the counterpart in the intrinsic frame $\{|\tau J K\rangle\}$ is a set of basis vectors with definite angular momentum quantum numbers $J$ and $K$.

The eigenstates of $\tilde{H}$ may be expressed as

$$
|\eta J M\rangle_{\mathrm{L}}=\sum_{\tau} a_{\tau}^{\eta}|\tau J M\rangle_{\mathrm{L}},
$$


where $a_{\tau}^{\eta}$ is the corresponding expansion coefficient, which should satisfy the eigen-equation

$$
\tilde{H}|\eta J M\rangle_{\mathrm{L}}=E_{J}^{\eta}|\eta J M\rangle_{\mathrm{L}}
$$

By using (16)-(20), the left side of (20) can be written as

$$
\tilde{H}|\eta J M\rangle_{\mathrm{L}}=\sum_{K} \hat{P}_{M K}^{J} \frac{1}{2 J+1} \sum_{\tau} a_{\tau}^{\eta} \hat{H}|\tau J K\rangle=\sum_{\tau \tau^{\prime} K} \frac{a_{\tau}^{\eta}}{2 J+1}\left|\tau^{\prime} J M\right\rangle_{\mathrm{L}}\left\langle\tau^{\prime} J K|\hat{H}| \tau J K\right\rangle,
$$

while the right side of (20) is

$$
E_{J}^{\eta}|\eta J M\rangle_{\mathrm{L}}=E_{J}^{\eta} \sum_{\tau^{\prime}} a_{\tau^{\prime}}^{\eta}\left|\tau^{\prime} J M\right\rangle_{\mathrm{L}}
$$

Combining (21) and (22), we get the eigen-equation in determining the eigen-energy and the corresponding expansion coefficients $a_{\tau}^{\eta}$ :

$$
\sum_{\tau} \sum_{K} \frac{1}{2 J+1}\left\langle\tau^{\prime} J K|\hat{H}| \tau J K\right\rangle a_{\tau}^{\eta}=E_{J}^{\eta} a_{\tau^{\prime}}^{\eta}
$$

in which the matrix element $\left\langle\tau^{\prime} J K|\hat{H}| \tau J K\right\rangle$ can be expressed in terms of those in the Nilsson plus pairing model as

$$
\begin{gathered}
\left\langle\tau^{\prime} J K|\hat{H}| \tau J K\right\rangle=\sum_{\xi \xi^{\prime}}\left\langle\tau^{\prime} J K \mid n, \xi\right\rangle\left\langle n, \xi|\hat{H}| n, \xi^{\prime}\right\rangle\left\langle n, \xi^{\prime} \mid \tau J K\right\rangle= \\
\sum_{\xi} \phi_{\xi}^{\tau^{\prime} J K} \phi_{\xi}^{\tau J K} E^{\xi},
\end{gathered}
$$

where $E^{\xi}$ is the eigenvalue of the Nilsson plus pairing model Hamiltonian, and $\phi_{\xi}^{\tau J K}=\langle n, \xi \mid \tau J K\rangle$ is the expansion coefficient, which can be chosen real and determined from the expansion:

$$
|\tau J K\rangle=\sum_{\xi}\langle n, \xi \mid \tau J K\rangle|n, \xi\rangle=\sum_{\xi} \phi_{\xi}^{\tau J K}|n, \xi\rangle
$$

There are many ways to determine the expansion coefficient $\phi_{\xi}^{\tau J K}$ in the intrinsic frame. One can adopt the angular momentum projection operator $[2,25]$

$$
P_{K}^{J}=P_{K K}^{J}=\frac{2 J+1}{8 \pi^{2}} \int d \alpha_{1} \sin \alpha_{2} d \alpha_{2} d \alpha_{3} D_{K K}^{J}\left(\alpha_{1}, \alpha_{2}, \alpha_{3}\right) e^{i \alpha_{1} \hat{J}_{z^{\prime}}} e^{i \alpha_{2} \hat{J}_{y^{\prime}}} e^{i \alpha_{3} \hat{J}_{z^{\prime}}}
$$

where $\hat{J}_{y^{\prime}}$ and $\hat{J}_{z^{\prime}}$ are the angular momentum operators in the intrinsic frame, and

$$
\begin{gathered}
D_{K K}^{J}\left(\alpha_{1}, \alpha_{2}, \alpha_{3}\right)=\sum_{k} \frac{(-)^{k}}{k !}\left(\begin{array}{c}
J-K \\
k
\end{array}\right)\left(\begin{array}{c}
J+K \\
k
\end{array}\right) e^{-i \alpha_{1} K}\left(\cos \alpha_{2} / 2\right)^{2 J-2 k}\left(\sin \alpha_{2} / 2\right)^{2 k} e^{-i \alpha_{3} K} \\
=e^{-i \alpha_{1} K} e^{-i \alpha_{3} K} d_{K K}^{J}\left(\alpha_{2}\right) .
\end{gathered}
$$

Generally, the resultant of the projection operator $P_{K}^{J}$ acting on the normalized orthogonal state $\left|\tau J^{\prime} K^{\prime}\right\rangle$ may be written as

$$
P_{K}^{J}\left|\tau J^{\prime} K^{\prime}\right\rangle=\lambda_{J} \delta_{J J^{\prime}} \delta_{K K^{\prime}}|\tau J K\rangle
$$

with $\lambda_{J}=1$. Acting with $P_{K}^{J}$ on $(25)$, we get

$$
P_{K}^{J}|\tau J K\rangle=|\tau J K\rangle=\sum_{\xi} \phi_{\xi}^{\tau J K} P_{K}^{J}|n, \xi\rangle .
$$


Then, acting with $\left\langle n, \xi^{\prime}\right|$ on (29), we obtain

$$
\lambda_{J} \phi_{\xi^{\prime}}^{\tau J K}=\sum_{\xi} \phi_{\xi}^{\tau J K}\left\langle n, \xi^{\prime}\left|P_{K}^{J}\right| n, \xi\right\rangle,
$$

which corresponds to an eigenvalue problem of the projection operator $P_{K}^{J}$ given by (26) under the subspace spanned by $\{|n, \xi\rangle\}$ with the eigenvalue $\lambda_{J}=1$, where

$$
\left\langle n, \xi^{\prime}\left|P_{K}^{J}\right| n, \xi\right\rangle=\frac{2 J+1}{2} \int_{0}^{\pi} \sin \beta d \beta d_{K K}^{J}(\beta)\left\langle n, \xi^{\prime}\left|e^{i \beta \hat{J}_{y^{\prime}}}\right| n, \xi\right\rangle .
$$

It is clear that $\left\{\phi_{\xi}^{\tau J K}\right\}$ are the $\tau$-th set of eigenvectors of the projection operator $P_{K}^{J}$. Because $\{|\tau J K\rangle\}$ are orthonormalized, $\left\{\phi_{\xi}^{\tau J K}\right\}$ should satisfy

$$
\sum_{\xi} \phi_{\xi}^{\tau J K} \phi_{\xi}^{\tau^{\prime} J^{\prime} K}=\delta_{\tau \tau^{\prime}} \delta_{J J^{\prime}}
$$

For the $i$-th Nilsson level, the Nilsson single-particle creation operator $a_{i}^{\dagger}$ can be expanded in terms of a set of intrinsic-frame creation operators in the spherical shell model with

$$
a_{i}^{\dagger}=\sum_{j_{i}} W_{j_{i}}^{i} c_{j_{i} \Omega_{i}}^{\dagger}, \quad a_{i}^{\dagger}=\sum_{j_{i}} W_{j_{i}}^{i}(-1)^{j_{i}-\Omega_{i}} c_{j_{i}}^{\dagger}-\Omega_{i},
$$

where $c_{j_{i} \Omega_{i}}^{\dagger}$ are the single-particle creation operators with definite angular momentum quantum number $j_{i}, \Omega_{i}$ is the projection of $j_{i}$ onto the third axis of the intrinsic frame, and $W_{j_{i}}^{i}$ is the normalized expansion coefficient when the $i$-th Nilsson state is expanded in terms of a set of the spherical shell model states, which is obtained from the Nilsson shell-model code. Therefore, the eigenstate $|n, \xi\rangle$ of the Nilsson mean-field plus pairing model Hamiltonian can be expressed as a product of a set of single-particle states of the spherical shell model. The set of state vectors $\{|\tau J K\rangle\}$ are also within the same shell-model subspace, so $|\tau J K\rangle$ can also be constructed from the $n$-particle states in the spherical shell model by using the angular momentum coupling, in which the additional quantum number $\tau$ is the multiplicity label needed for the angular momentum coupling $j_{1} \otimes j_{2} \otimes \cdots \otimes j_{n} \downarrow J$, which can be determined efficiently by using the polynomial approach outlined in [37]. Thus, both the eigenstates $\{|n, \xi\rangle\}$ of the Nilsson mean-field plus pairing model Hamiltonian and the $n$-particle state vectors with total angular momentum $J,\{|\tau J K\rangle\}$, can all be expressed in terms of a linear combination of products of the single-particle states of the spherical shell model, with which the overlaps $\phi_{\xi}^{\tau J K}=\langle n, \xi \mid \tau J K\rangle$ can easily be calculated. Once the overlaps $\left\{\phi_{\xi}^{\tau J K}\right\}$ are obtained, the eigenequation (23) simultaneously determines, after the angular momentum projection, the excitation energies $\left\{E_{J}^{\eta}\right\}$ and the corresponding eigenstates $\left\{|\eta J M\rangle_{\mathrm{L}}\right\}$.

Moreover, in the previous studies $[25,36]$, it was not necessary to show the relation of $|\tau J M\rangle_{\mathrm{L}}$ with the corresponding intrinsic state $|\tau J K\rangle$ because the Hamiltonian used in previous work is rotational invariant, namely, in this case, the results of diagonalizing the rotational invariant Hamiltonian in the laboratory frame $|\tau J M\rangle_{\mathrm{L}}$ and those obtained from the intrinsic frame is the same. Actually, it is well known from textbooks, e. g. [38], that a state in the laboratory frame with good angular momentum quantum number $J$ and $M$ can be expressed in terms of those in the intrinsic frame $|\tau J K\rangle$ as

$$
|\tau J M\rangle_{\mathrm{L}}=\sqrt{\frac{1}{8 \pi^{2}}} \sum_{K} D_{M K}^{J *}(\alpha, \beta, \gamma)|\tau J K\rangle
$$

for given Euler angles $\alpha, \beta$, and $\gamma$, which is orthogonal with respect to the quantum numbers $\eta$, $J$, and $M$ :

$$
{ }_{\mathrm{L}}\left\langle\tau^{\prime} J^{\prime} M^{\prime} \mid \tau J M\right\rangle_{\mathrm{L}}=\delta_{\tau \tau^{\prime}} \delta_{J J^{\prime}} \delta_{M M^{\prime}},
$$

where the well-known orthogonal relation

$$
\frac{2 J+1}{8 \pi^{2}} \int_{0}^{2 \pi} d \alpha \int_{0}^{\pi} \sin \beta d \beta \int_{0}^{2 \pi} d \gamma D_{M^{\prime} K^{\prime}}^{J^{\prime}}(\alpha, \beta, \gamma) D_{M K}^{J *}(\alpha, \beta, \gamma)=\delta_{J J^{\prime}} \delta_{M M^{\prime}} \delta_{K K^{\prime}}
$$


is used. In this case, only pure (fixed point) rotation of a nucleus is assumed. Since the Euler angles $\alpha, \beta$, and $\gamma$ are not fixed, integration over the Euler angles should be preformed in calculating matrix elements of operators in the laboratory frame, especially in calculating quadrupole moments, B(E2) values, etc.

Moreover, it can easily be proven that the projected Hamiltonian $\tilde{H}$ is rotational invariant,

$$
\tilde{H}=R \tilde{H} R^{-1} \quad \forall R \in R_{3},
$$

by using the identity

$$
\sum_{M} D_{M_{1} M}^{J}(R) D_{M_{2} M}^{J *}(R)=\delta_{M_{1} M_{2}} \forall R \in R_{3}
$$

As is well known, any operator $\hat{T}$ in the laboratory frame can be expressed in terms of those in the intrinsic frame $\hat{T}($ in) as

$$
\hat{T}=\rho R \hat{T}(\text { in }) R^{-1},
$$

where $R \in R_{3}, \rho$ is a scalar factor discussed in the Appendix B. Thus, irreducible tensor operators $T_{\mu}^{k}$ in the laboratory frame are related to those in the intrinsic frame by

$$
T_{\mu}^{(k)}=\rho_{k} \sum_{\nu} D_{\mu \nu}^{k *}(\alpha, \beta, \gamma) T_{\nu}^{(k)}(\text { in })
$$

where $T_{\nu}^{k}$ (in) are the intrinsic tensor operators, and $\rho_{k}$ is a scalar factor to ensure the $R_{3}$-reduced matrix elements of $T_{\mu}^{k}$ in the laboratory frame are the same as the corresponding ones in the intrinsic frame, which is determined in Appendix B. For example, the quadrupole operator $Q_{\mu}^{(2)}$ in the laboratory frame can be expressed as

$$
Q_{\mu}^{(2)}=\rho_{2}\left(R Q^{(2)}(\mathrm{in}) \mathrm{R}^{-1}\right)_{\mu}=\rho_{2} \sum_{\nu} D_{\mu \nu}^{2 *}(\alpha, \beta, \gamma) Q_{\nu}^{(2)}(\mathrm{in})
$$

where $Q_{\nu}^{(2)}\left(\right.$ in) is the quadrupole operator in the intrinsic frame. As shown in Appendix B, $\rho_{2}=5$ for the exact angular momentum projection, while $\rho_{2}=3$ is set for the approximate angular momentum projection discussed in the following.

In the Nilsson mean-field plus pairing model, the Nilsson potential is assumed axially deformed. The intrinsic quadrupole operator is given by

$$
\hat{Q}_{\mu}^{(2)}(\mathrm{in})=\delta_{\mu 0} \sum_{t=1}^{n} r_{t}^{2} Y_{2 \mu}\left(\theta_{t}, \varphi_{t}\right),
$$

where the sum runs over all valence nucleons considered. Within a single harmonic oscillator major shell with the principal quantum number $N_{\mathrm{HO}}, \hat{Q}_{0}^{(2)}(\mathrm{in})$ can conveniently be written in the spherical shell model basis as

$$
\hat{Q}_{0}^{(2)}(\mathrm{in})=\sum_{j j^{\prime}} q\left(j j^{\prime}\right)\left(c_{j}^{\dagger} \tilde{c}_{j^{\prime}}\right)_{0}^{(2)},
$$

where

$$
q\left(j j^{\prime}\right)=\frac{(-1)^{j-\frac{1}{2}}}{\sqrt{20 \pi}} \sqrt{(2 j+1)\left(2 j^{\prime}+1\right)}\left\langle j \frac{1}{2}, j^{\prime}-\frac{1}{2} \mid 20\right\rangle\left\langle N_{\mathrm{HO}} l\left|r^{2}\right| N_{\mathrm{HO}} l^{\prime}\right\rangle,
$$

in which $\left\langle j \frac{1}{2}, j^{\prime}-\frac{1}{2} \mid 20\right\rangle$ is the CG coefficient, and

$$
\begin{array}{r}
\left\langle N_{\mathrm{HO}} l^{\prime}\left|r^{2}\right| N_{\mathrm{HO}} l\right\rangle=\sqrt{\left(N_{\mathrm{HO}}-l+2\right)\left(N_{\mathrm{HO}}+l+1\right)} b^{2} \delta_{l^{\prime} l-2}+ \\
\left(N_{\mathrm{HO}}+3 / 2\right) b^{2} \delta_{l^{\prime} l}+\sqrt{\left(N_{\mathrm{HO}}-l\right)\left(N_{\mathrm{HO}}+l+3\right)} b^{2} \delta_{l^{\prime} l+2},
\end{array}
$$


where $b=\sqrt{\hbar / M_{n} \omega_{0}}$, in which $M_{n}$ is the nucleon mass, and the values of $\hbar \omega_{0}$ in the Nilsson shell model are provided in Appendix A. Thus, the electric quadrupole moment of the state $|\eta J M=J\rangle_{\mathrm{L}}$ is given by

$$
\begin{gathered}
Q_{J}^{\eta}=\sqrt{\frac{16 \pi}{5}}{ }_{\mathrm{L}}\left\langle\eta J J\left|Q_{0}^{(2)}(\mathrm{E})\right| \eta J J\right\rangle_{\mathrm{L}}= \\
\sqrt{\frac{16 \pi}{5}}\langle J J, 20 \mid J J\rangle\left\langle\eta J\left\|\left(q_{2}^{(\pi)} Q^{(2)}(\mathrm{in} ; \pi)+q_{2}^{(\nu)} Q^{(2)}(\mathrm{in} ; \nu)\right)\right\| \eta J\right\rangle,
\end{gathered}
$$

where the integration over the Euler angles has been preformed, $Q_{\mu}^{(2)}(\mathrm{E})=q_{2}^{(\pi)} Q_{\mu}^{(2)}+q_{2}^{(\nu)} Q_{\mu}^{(2)}$ when both the proton and the neutron sectors are considered, in which $q_{2}^{(\pi)}$ and $q_{2}^{(\nu)}$ are the effective charge of valence protons and that of neutrons, respectively, and $|\eta J K\rangle=\sum_{\tau} a_{\tau}^{\eta}|\tau J K\rangle$ is a linear combination of the intrinsic states $\{|\tau J K\rangle\}$. Similarly, the $B(\mathrm{E} 2)$ value is calculated by

$$
\begin{gathered}
B\left(\mathrm{E} 2 ; \eta J_{i} \rightarrow \eta^{\prime} J_{f}\right)=\frac{2 J_{f}+1}{2 J_{i}+1}\left|\mathrm{~L}\left\langle\eta^{\prime} J_{f}\left\|Q^{(2)}(\mathrm{E})\right\| \eta J_{i}\right\rangle_{\mathrm{L}}\right|^{2} \\
=\frac{2 J_{f}+1}{2 J_{i}+1}\left|\left\langle\eta^{\prime} J_{f}\left\|\left(q_{2}^{(\pi)} Q^{(2)}(\mathrm{in} ; \pi)+q_{2}^{(\nu)} Q^{(2)}(\mathrm{in} ; \nu)\right)\right\| \eta J_{i}\right\rangle\right|^{2} .
\end{gathered}
$$

It is clear that $Q_{J}^{\eta}$ and $\mathrm{B}(\mathrm{E} 2)$ values are exactly the same as the corresponding ones in the intrinsic frame when $\rho_{2}=5$ is set. It is obvious that the angular momentum projection reorganizes the intrinsic states into a physical state with good angular momentum only, which keeps physical quantities determined by the dynamics not related to the angular momentum in the intrinsic frame unchanged. In contrast to the angular momentum projection carried out in [25, 36], in which the intrinsic state used is fixed from the HF solutions, or a few deformed basis vectors of the Nilsson plus BCS are considered in the calculation as approximations, all intrinsic states in the deformed mean-field plus pairing model should be considered in the exact angular momentum projection in this work in order to obtain eigenstates of the system with good angular momentum.

(2) An approximate angular momentum projection. In the axially symmetric Nilsson plus pairing model, $K$ is a good quantum number. Lower-lying excitations are dominated by $K=0$ components with axial symmetry, while $K= \pm 1, \pm 2, \cdots$ components are still possible for higher excited states with more than one broken pairs. Therefore, these $K \neq 0$ components may be neglected in the lower energy regime as an approximation. With such approximation, the dimension of the subspace in diagonalizing the Hamiltonian is greatly reduced. However, eigen-energies with $J \neq 0$ will become a little different in comparison to the results obtained from the exact angular momentum projection, which will be discussed in the next subsection.

The projected Hamiltonian in the approximate angular momentum projection, restricted to $K=0$, may be written as

$$
\tilde{H}_{0}=\sum_{J M} P_{M 0}^{J} \hat{H} P_{0 M}^{J}
$$

where

$$
P_{M 0}^{J}=\sum_{\tau}|\tau J M\rangle_{\mathrm{L}}\left\langle\tau J K=0\left|, \quad P_{0 M}^{J}=\left(P_{M 0}^{J}\right)^{\dagger}=\sum_{\tau}\right| \tau J K=0\right\rangle_{\mathrm{L}}\langle\tau J M|
$$

according to (17). The eigenstates of (48) are thus given by

$$
|\eta J M\rangle_{\mathrm{L}}=\sqrt{\frac{2 J+1}{8 \pi^{2}}} D_{M 0}^{J *}(\alpha, \beta, \gamma)|\eta J 0\rangle,
$$

in which

$$
|\eta J 0\rangle=\sum_{\tau} \bar{a}_{\tau}^{\eta}|\tau J 0\rangle
$$

is a linear combination of intrinsic states with $K=0$, where $\bar{a}_{\tau}^{\eta}$ is the corresponding expansion coefficient, which should satisfy the eigen-equation

$$
\sum_{\tau}\left\langle\tau^{\prime} J 0|\hat{H}| \tau J 0\right\rangle \bar{a}_{\tau}^{\eta}=\bar{E}_{J}^{\eta} \bar{a}_{\tau^{\prime}}^{\eta}
$$


where $\bar{E}_{J}^{\eta}$ is the corresponding eigen-energy of (48):

$$
\tilde{H}_{0}|\eta J M\rangle_{\mathrm{L}}=\bar{E}_{J}^{\eta}|\eta J M\rangle_{\mathrm{L}}
$$

Similar to (37), one can directly prove that the Hamiltonian (48) is also rotational invariant, even though the eigenvalues of (48) are approximations to those of (37) due to the fact that only intrinsic states with $K=0$ are taken in the projection. Tough the approximation with $K=0$ intrinsic states breaks the rotation covariance, the eigenstates (50) may be regarded as an approximation with higher excited intrinsic states neglected.

The electric quadrupole moment of the state $|\eta J M=J\rangle_{\mathrm{L}}$ can be simplified as

$$
\begin{gathered}
Q_{J}^{\eta}=\sqrt{\frac{16 \pi}{5}}{ }_{\mathrm{L}}\left\langle\eta J J\left|Q_{0}^{(2)}(\mathrm{E})\right| \eta J J\right\rangle_{\mathrm{L}}= \\
\sqrt{\frac{16 \pi}{5}} \rho_{2}\langle J J, 20 \mid J J\rangle\langle J 0,20 \mid J 0\rangle\left\langle\eta J 0\left|\left(q_{2}^{(\pi)} Q_{0}^{(2)}(\mathrm{in} ; \pi)+q_{2}^{(\nu)} Q_{0}^{(2)}(\mathrm{in} ; \nu)\right)\right| \eta J 0\right\rangle,
\end{gathered}
$$

while the $B(\mathrm{E} 2)$ value is given by

$$
\begin{gathered}
B\left(\mathrm{E} 2 ; \eta J_{i} \rightarrow \eta^{\prime} J_{f}\right)=\frac{2 J_{f}+1}{2 J_{i}+1}\left|{ }_{\mathrm{L}}\left\langle\eta^{\prime} J_{f}|| Q^{(2)}(\mathrm{E}) \| \eta J_{i}\right\rangle_{\mathrm{L}}\right|^{2} \\
=\left(\rho_{2}\left\langle J_{i} 0,20 \mid J_{f} 0\right\rangle\left\langle\eta^{\prime} J_{f} 0\left|\left(q_{2}^{(\pi)} Q_{0}^{(2)}(\mathrm{in} ; \pi)+q_{2}^{(\nu)} Q_{0}^{(2)}(\mathrm{in} ; \nu)\right)\right| \eta J_{i} 0\right\rangle\right)^{2} .
\end{gathered}
$$

As shown in Appendix B, $\rho_{2}=3$ is set for this case.

(3) Comparison of the results obtained from the exact and approximate projections. In order to elucidate the angular momentum projection outlined in the previous subsection, we only consider simple cases with two valence protons or neutrons within the $d s$-shell. For two valence particles in the $d s$-shell, there are 66 independent two-particle states, in which there are only 14 of them with $K=0$. In the deformed Nilsson shell model, the 6 Nilsson singleparticle states are expressed as $a_{i \Omega_{i}}^{\dagger}$ with $i=1,2, \cdots, 6$, of which $\Omega_{1}=\Omega_{4}=\Omega_{5}=\frac{1}{2}, \Omega_{2}=\Omega_{6}=\frac{3}{2}$, and $\Omega_{3}=\frac{5}{2}$ are assigned. The 14 two-particle states with $K=0$ in the Nilsson model are listed in Table I. Equivalently, in the spherical shell model basis, there are also $14 K=0$ states explicitly shown in Table II.

TABLE I: Two-particle states with $K=0$ in the deformed Nilsson shell model.

\begin{tabular}{l}
\hline \hline$\{2, \overline{6}\}\rangle=a_{2, \frac{3}{2}}^{\dagger} a_{\overline{6},-\frac{3}{2}}^{\dagger}|0\rangle,|\{6, \overline{2}\}\rangle=a_{6, \frac{3}{2}}^{\dagger} a_{\overline{2},-\frac{3}{2}}^{\dagger}|0\rangle,|\{1, \overline{4}\}\rangle=a_{1, \frac{1}{2}}^{\dagger} a_{\overline{4},-\frac{1}{2}}^{\dagger}|0\rangle,|\{4, \overline{1}\}\rangle=a_{4, \frac{1}{2}}^{\dagger} a_{\overline{1},-\frac{1}{2}}^{\dagger}|0\rangle$, \\
$|\{4, \overline{5}\}\rangle=a_{4, \frac{1}{2}}^{\dagger} a_{\overline{5},-\frac{1}{2}}^{\dagger}|0\rangle,|\{5, \overline{4}\}\rangle=a_{5, \frac{1}{2}}^{\dagger} a_{\overline{4},-\frac{1}{2}}^{\dagger}|0\rangle,|\{1, \overline{5}\}\rangle=a_{1, \frac{1}{2}}^{\dagger} a_{\overline{5},-\frac{1}{2}}^{\dagger}|0\rangle,|\{5, \overline{1}\}\rangle=a_{5, \frac{1}{2}}^{\dagger} a_{\overline{1},-\frac{1}{2}}^{\dagger}|0\rangle$, \\
$|\{1, \overline{1}\}\rangle=a_{1, \frac{1}{2}}^{\dagger} a_{\overline{1},-\frac{1}{2}}^{\dagger}|0\rangle,|\{2, \overline{2}\}\rangle=a_{2, \frac{1}{2}}^{\dagger} a_{\overline{2},-\frac{1}{2}}^{\dagger}|0\rangle,|\{3, \overline{3}\}\rangle=a_{3, \frac{5}{2}}^{\dagger} a_{\overline{3},-\frac{5}{2}}^{\dagger}|0\rangle,|\{4, \overline{4}\}\rangle=a_{4, \frac{1}{2}}^{\dagger} a_{\overline{4},-\frac{1}{2}}^{\dagger}|0\rangle$, \\
$|\{5, \overline{5}\}\rangle=a_{5, \frac{1}{2}}^{\dagger} a_{\overline{5},-\frac{1}{2}}^{\dagger}|0\rangle,|\{6, \overline{6}\}\rangle=a_{6, \frac{3}{2}}^{\dagger} a_{\overline{6},-\frac{3}{2}}^{\dagger}|0\rangle$.
\end{tabular}

By using the explicit results of two-particle states with good angular momentum similar to those shown in Table II and those in the Nilsson basis similar to those shown in Table I, together with the expansion coefficients $W_{j}^{i}$ for the $i$-th single-particle state $a_{i \Omega_{i}}^{\dagger}$ in the Nilsson model expanded in terms of $c_{j \Omega_{i}}^{\dagger}$ in the spherical shell model according to Eq. (33), the overlaps $\phi_{\xi}^{\tau J K}$ can easily be evaluated.

Since the ordering of level energies of the first $2^{+}$and $4^{+}$states and the related energy gaps are most important in the spectrum, these two energy levels as functions of the pairing interaction strength obtained from the Nilsson meanfield plus pairing model with both the exact and approximate angular momentum projection are calculated for both the MSP and NLP cases. For the spherical mean-field plus the standard pairing case with $G_{i}=0$ for $i=1,2, \cdots, 6$, as shown in panels (a) and (b) of Fig. 1, level energies of the first $2^{+}$state and the first $4^{+}$state are degenerate after the exact or approximate angular momentum projection is applied to the model. The results in both cases are the same or very close, which justifies the validity of the angular momentum projection procedure. To avoid such degeneracy for describing realistic nuclei, instead of the standard pairing interaction, one may consider the MSP. In the $d s$-shell for example, if we set $G_{i j}=G$ for $i \neq j$ and $G_{i i}$ with different values for different level $i$, the degeneracy of the first $2^{+}$and $4^{+}$states can be avoided effectively. Panels (c) and (d) of Fig. 1 show level energies of the first $2^{+}$ state and the first $4^{+}$state as functions of $G$ with $G_{33}=2 G$ and $G_{i j}=G$ when $i \neq j$ or $i=j \neq 3$, that are obtained 
from the exact and approximate angular momentum projections, respectively. It can be observed that the energy gap between $2_{1}^{+}$and $4_{1}^{+}$increases with increasing $G$ in this case. The gap becomes larger in the approximate projection, but still keeps the pattern of the exact projection, which shows that the approximate angular momentum projection seems acceptable for comparatively small pairing strengths. In the deformed mean-field case, similar to the spherical mean-field case shown in panel (a) of Fig. 1, the level energies of the first $2^{+}$state and the first $4^{+}$state are also degenerate. In order to avoid the degeneracy, the MSP may be adopted. Panel (a) and (b) of Fig. 2 provide level energies of the first $2^{+}$state and the first $4^{+}$state as functions of $G$ (in MeV) obtained from the deformed Nilsson mean-field plus the MSP with $G_{33}=2 G$ and $G_{i j}=G$ when $i \neq j$ or $i=j \neq 3$ after the exact and approximate projections, respectively. Although deviations in the level energies are comparatively small, the ordering of these two levels is altered in the approximate projection when the pairing interaction strength is small. Nevertheless, the ordering of these two levels becomes valid when the pairing interaction strength is large enough. The situation with the NLP is similar. As shown in panel (c) and (d) of Fig. 2, the ordering of these two levels in the approximate case becomes the same as that obtained in the exact projection when the pairing interaction strength $A \geq 0.62 \mathrm{MeV}$, where $A_{i i}=A$ are taken for any $i$. However, in this case, the $2_{1}^{+}$level is lower than that obtained from the exact projection due to the approximation. Moreover, it can be observed that these level energies produced from the NLP are lower in energy when $A \sim G$, which is mainly due to the fact that the pairing interactions beyond the nearest level are neglected. In order to produce the same value of these level energies as those in the MSP case, larger nearest pairing interaction strength $A$ is needed.

TABLE II: Two-particle intrinsic states with angular momentum quantum number $J$ and $K=0$.

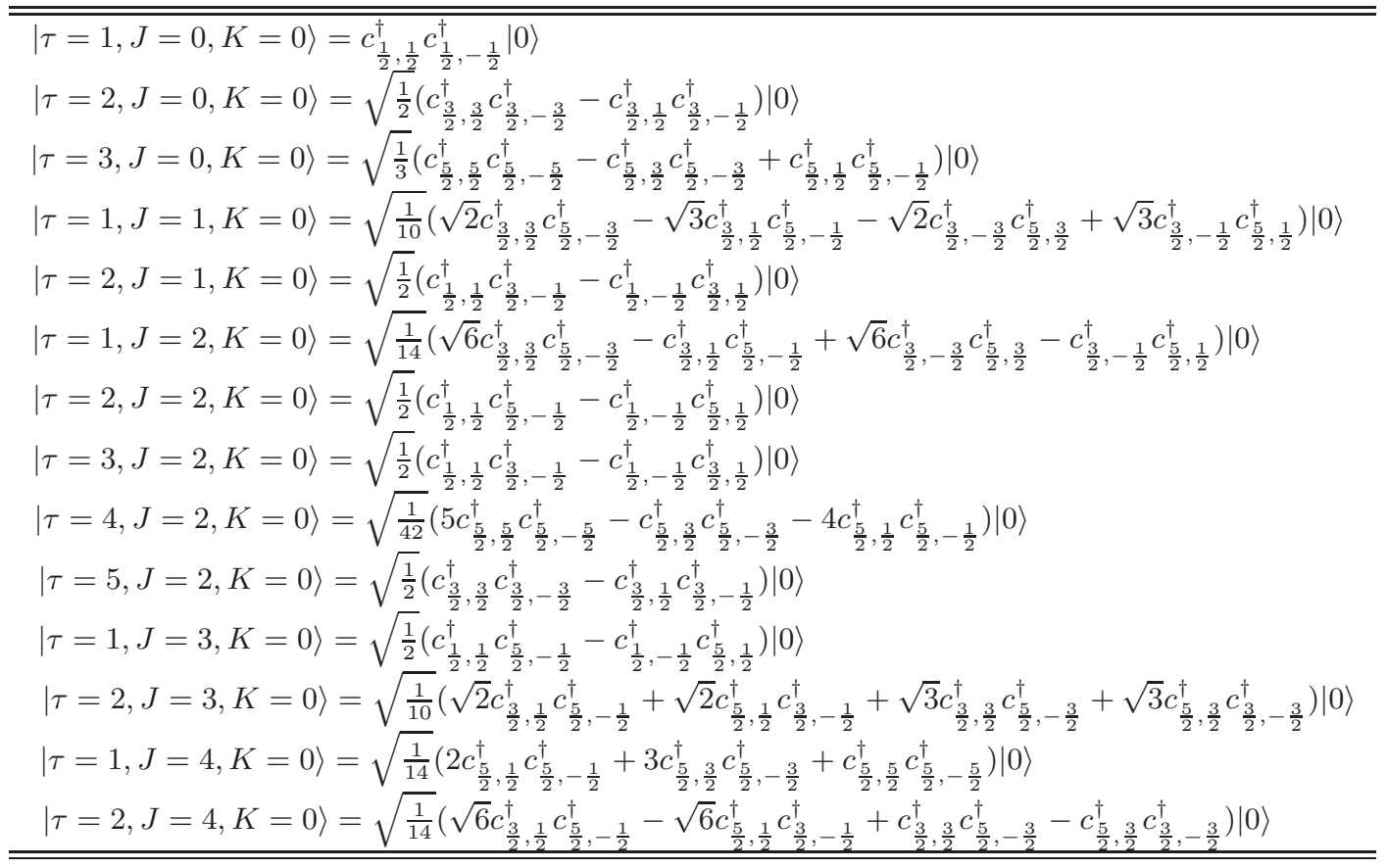



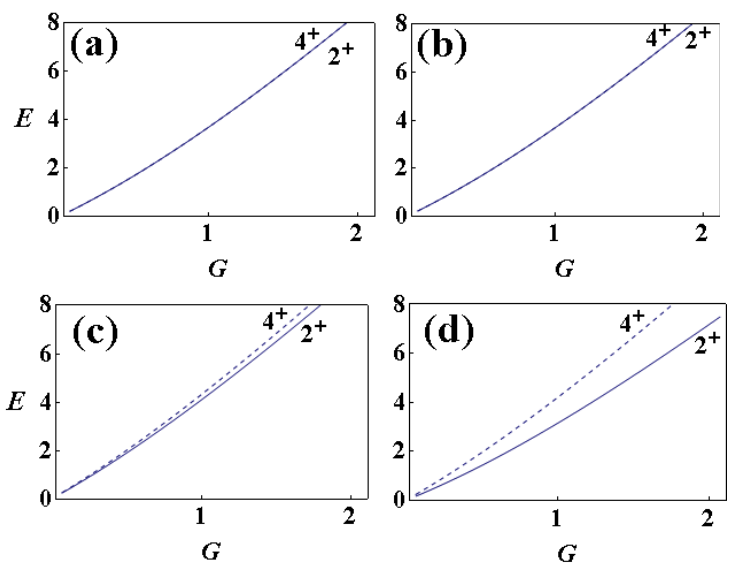

FIG. 1: Level energies (in $\mathrm{MeV}$ ) of the first $2^{+}$state and the first $4^{+}$state as functions of $G$ (in MeV). The results are obtained from the spherical mean-field in the $d s$-shell with single-particle energies calculated from the axially deformed Nilsson shell model with deformation parameter $\epsilon_{2}=0$, from which we get $\varepsilon_{1}=\varepsilon_{2}=\varepsilon_{3}=-1.08182 \mathrm{MeV}, \varepsilon_{4}=2.35977 \mathrm{MeV}$, $\varepsilon_{5}=\varepsilon_{6}=7.52214 \mathrm{MeV}$. (a) Results of the standard pairing obtained from the exact projection; (b) The same as (a) but from the approximate projection; (c) Results of the MSP with $G_{33}=2 G$ and $G_{i j}=G$ when $i \neq j$ or $i=j \neq 3$ from the exact projection; (d) The same as (c) but from the approximate projection.
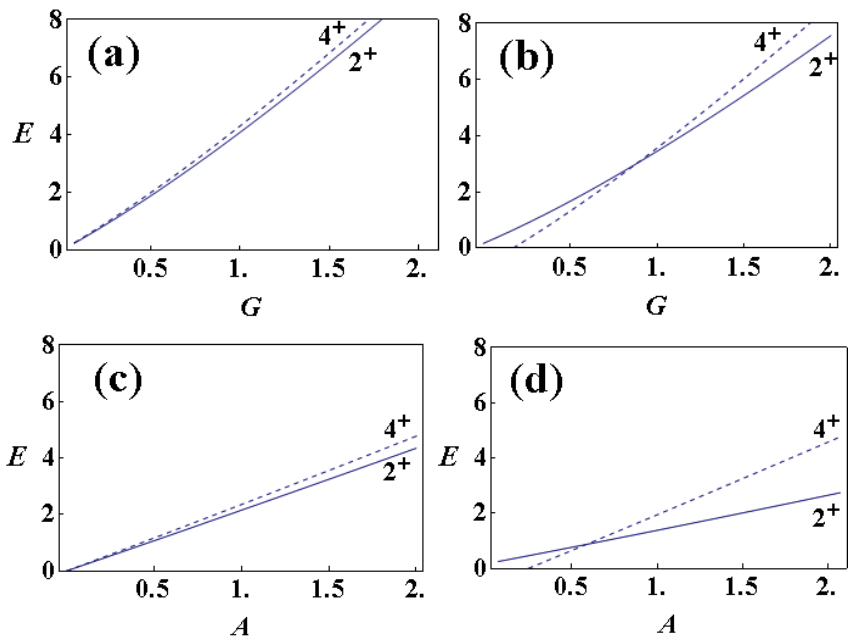

FIG. 2: Level energies (in MeV) of the first $2^{+}$state and the first $4^{+}$state as functions of $G$ (in MeV) with the MSP or as functions of $A$ (in $\mathrm{MeV}$ ) with the NLP, in which $A_{i i}=A$ are taken for any $i$ and $B=0.1\left(\mathrm{MeV}^{-2}\right)$ is taken. The results are obtained from the deformed mean-field in the $d s$-shell with single-particle energies calculated from the axially deformed Nilsson shell model with deformation parameter $\epsilon_{2}=0.1$, from which we get $\varepsilon_{1}=-2.23049 \mathrm{MeV}, \varepsilon_{2}=-1.28 \mathrm{MeV}, \varepsilon_{3}=0.07729 \mathrm{MeV}$, $\varepsilon_{4}=2.55610 \mathrm{MeV}, \varepsilon_{5}=7.02966 \mathrm{MeV}$, and $\varepsilon_{6}=8.39782 \mathrm{MeV}$. (a) Results of the MSP with $G_{33}=2 G$ and $G_{i j}=G$ when $i \neq j$ or $i=j \neq 3$ obtained from the exact projection; (b) The same as (a) but from the approximate projection; (c) Results of the NLP; (d) The same as (c) but from the approximate projection.

\section{APPLiCATION TO ${ }^{18} \mathrm{O},{ }^{18} \mathrm{Ne},{ }^{20} \mathrm{Ne}$, AND ${ }^{24} \mathrm{Mg}$}

As shown in the previous section, the dimension of the $K=0$ intrinsic subspace is greatly reduced when the approximate angular momentum projection only including $K=0$ intrinsic states is considered, which becomes more obvious when more valence particles are involved. More importantly, the approximate angular momentum projection seems acceptable for a reasonable range of the pairing strength parameters in the axially deformed Nilsson meanfield plus the pairing model when the MSP or the NLP is adopted. In order to justify that the approximate angular 
momentum projection is indeed acceptable for describing realistic nuclear systems, the axially deformed Nilsson meanfield plus the pairing models with both the exact and approximate angular momentum projection are applied to ${ }^{18} \mathrm{O}$ and ${ }^{18} \mathrm{Ne}$, while only the approximate projection is used to describe ${ }^{20} \mathrm{Ne}$ and ${ }^{24} \mathrm{Mg}$.

In this work, the deformation parameter $\epsilon_{2}$ used in the Nilsson shell model for each nucleus is taken from [39] determined systematically from the corresponding experimental data [40], from which we get $\epsilon_{2}=0.017$ for ${ }^{18} \mathrm{O}$, $\epsilon_{2}=0.1$ for ${ }^{18} \mathrm{Ne}, \epsilon_{2}=0.3$ for ${ }^{20} \mathrm{Ne}$, and $\epsilon_{2}=0.333$ for ${ }^{24} \mathrm{Mg}$, respectively. With these deformation parameters, the single-particle energies for these nuclei are obtained from the axially deformed Nilsson model with other paramters shown in the Appendix A. Therefore, the single-particle energies are thus fixed from the Nilsson model. The only adjustable parameters are pairing interaction strengthes. It is shown that the pairing interactions within the same Nilsson levels with $G_{66} \geq G_{55} \geq \cdots \geq G_{11}$ in the MSP (MSP0) or with $A_{66} \geq A_{55} \geq \cdots \geq A_{11}$ in the NLP (NLP0) are necessary to avoid level degeneracy. $G_{11}=G_{22}=G_{1}<G_{33}=G_{44}=G_{2}<G_{55}=G_{66}=G_{3}$ or $A_{11}=A_{22}=A_{1}<A_{33}=A_{44}=A_{2}<A_{55}=A_{66}=A_{3}$ is chosen in the MSP or NLP, and the pairing interactions among neutrons and protons are chosen to be the same in order to keep the number of free parameters as few as possible, accept for ${ }^{20} \mathrm{Ne}$ and ${ }^{24} \mathrm{Mg}$, in which $G_{11}^{\pi} \neq G_{11}^{\nu}$ and $G_{22}^{\pi} \neq G_{22}^{\nu}$ or $A_{22}^{\pi} \neq A_{22}^{\nu}$ are used in order to avoid the additional $S_{2}$ proton-neutron permutation symmetry. Thus, there are 4 parameters in the MSP and 5 parameters in the NLP accept for ${ }^{20} \mathrm{Ne}$ and ${ }^{24} \mathrm{Mg}$, of which the choice of the parameters will be shown separately. In comparison with the SU(3) shell model, besides the shell model inputs, there are also 4-5 additional parameters used in [41-43] in order to describe low-lying states of ${ }^{20} \mathrm{Ne}$.

For ${ }^{18} \mathrm{O}\left({ }^{18} \mathrm{Ne}\right)$, we only consider two valence neutrons (protons) in the deformed $d s$-shell with either the MSP (MSP0) or the NLP (NLP0) in the exact (approximate) angular momentum projection, respectively. Therefore, only positive parity states are involved. The corresponding Nilsson single-particle energies derived in the deformed Nilsson model, as well as adjustable parameters in the MSP, and those in the NLP are listed in Table III, while adjustable parameters in the MSP0, and those in the NLP0 are listed in Table IV. The deviation between the final theoretical results and the corresponding experimental values is measured by a mean-square deviation defined as

$$
\chi=\left[\frac{1}{\mathcal{N}} \sum_{\mu}\left(X_{\mu}^{\mathrm{Exp}}-X_{\mu}^{\mathrm{Th}}\right)^{2}\right]^{1 / 2}
$$

where $X_{\mu}$ stands for a level energy (in $\mathrm{MeV}$ ) or a B(E2) value (in W.u.), $X_{\mu}^{\text {Exp }}$ is the experimental value, $X_{\mu}^{\text {Th }}$ is the corresponding theoretical value, and $\mathcal{N}$ is the total number of levels or $\mathrm{B}(\mathrm{E} 2)$ values calculated.

TABLE III: Single-particle energies (in $\mathrm{MeV}$ ) within the $d s$ shell generated from the deformed Nilsson shell model for ${ }^{18} \mathrm{O}$, ${ }^{18} \mathrm{Ne}$, and ${ }^{20} \mathrm{Ne}$, as well as adjustable parameters $G_{i}$ and $G$ (in MeV) in the modified standard pairing (MSP), or $A_{i}$ and $A$ in $(\mathrm{MeV})$ in the nearest-level pairing (NLP) for ${ }^{18} \mathrm{O}$ and ${ }^{18} \mathrm{Ne}$ used in the exact angular momentum projection. In the NLP, the parameter $B=0.1\left(\mathrm{MeV}^{-2}\right)$ is taken.

\begin{tabular}{|c|c|c|}
\hline${ }^{18} \mathrm{O}$ & ${ }^{18} \mathrm{Ne}$ & ${ }^{20} \mathrm{Ne}$ \\
\hline$\varepsilon_{1}^{(\nu)}=-1.24041$ & $\varepsilon_{1}^{(\pi)}=-2.23049$ & $\varepsilon_{1}^{(\nu)}=\varepsilon_{1}^{(\pi)}=-8.55737$ \\
\hline$\varepsilon_{2}^{(\nu)}=-1.11854$ & $\varepsilon_{2}^{(\pi)}=-1.28000$ & $\varepsilon_{2}^{(\nu)}=\varepsilon_{2}^{(\pi)}=-5.08317$ \\
\hline$\varepsilon_{3}^{(\nu)}=-0.89423$ & $\varepsilon_{3}^{(\pi)}=0.07729$ & $\varepsilon_{3}^{(\nu)}=\varepsilon_{3}^{(\pi)}=-1.06761$ \\
\hline$\varepsilon_{4}^{(\nu)}=2.36780$ & $\varepsilon_{4}^{(\pi)}=2.55610$ & $\varepsilon_{4}^{(\nu)}=\varepsilon_{4}^{(\pi)}=2.50000$ \\
\hline$\varepsilon_{5}^{(\nu)}=7.39963$ & $\varepsilon_{5}^{(\pi)}=7.02966$ & $\varepsilon_{5}^{(\nu)}=\varepsilon_{5}^{(\pi)}=3.59920$ \\
\hline$\varepsilon_{6}^{(\nu)}=7.65543$ & $\varepsilon_{6}^{(\pi)}=8.39782$ & $\varepsilon_{6}^{(\nu)}=\varepsilon_{6}^{(\pi)}=6.33528$ \\
\hline$G_{1}^{(\nu)}=0.01 G \quad A_{1}^{(\nu)}=0.1 A$ & $G_{1}^{(\pi)}=0.1 G \quad A_{1}^{(\pi)}=0.1 A^{(\pi)}$ & \\
\hline$G_{2}^{(\nu)}=23 G \quad A_{2}^{(\nu)}=1.5 A$ & $G_{2}^{(\pi)}=8 G \quad A_{2}^{(\pi)}=3 A^{(\pi)}$ & \\
\hline$G_{3}^{(\nu)}=55 G \quad A_{3}^{(\nu)}=15 A$ & $G_{3}^{(\pi)}=30 G^{(\pi)} \quad A_{3}^{(\pi)}=15 A^{(\pi)}$ & \\
\hline$G^{(\nu)}=0.25 \quad A_{3}^{(\nu)}=1.0$ & $G^{(\pi)}=0.5 \quad A^{(\pi)}=1.0$ & \\
\hline
\end{tabular}


TABLE IV: Adjustable parameters $G_{i}$ and $G$ (in MeV) in the modified standard pairing (MSP0), or $A_{i}$ and $A$ in $(\mathrm{MeV})$ in the nearest-level pairing (NLP0) for ${ }^{18} \mathrm{O},{ }^{18} \mathrm{Ne}$, and ${ }^{20} \mathrm{Ne}$ (see text) used in the approximate angular momentum projection. In the NLP0, the parameter $B=0.1\left(\mathrm{MeV}^{-2}\right)$ is taken.

\begin{tabular}{ccccc}
\hline \multicolumn{2}{c}{${ }^{18} \mathrm{O}$} & \multicolumn{2}{c}{${ }^{18} \mathrm{Ne}$} & ${ }^{20} \mathrm{Ne}$ \\
\hline$G_{1}^{(\nu)}=0.01 G$ & $A_{1}^{(\nu)}=1 A$ & $G_{1}^{(\pi)}=0.1 G^{(\pi)}$ & $A_{1}^{(\pi)}=0.1 A^{(\pi)}$ & $G_{11}^{(\pi)}=0.3 G \quad G_{11}^{(\nu)}=0$ \\
$G_{2}^{(\nu)}=6 G \quad A_{2}^{(\nu)}=2.5 A$ & $G_{2}^{(\pi)}=10 G^{(\pi)}$ & $A_{22}^{(\pi)}=3 A^{(\pi)}$ & $G_{22}^{(\pi)}=4.2 G \quad G_{22}^{(\nu)}=4.0 G$ \\
$G_{3}^{(\nu)}=29 G$ & $A_{3}^{(\nu)}=15 A$ & $G_{3}^{(\pi)}=20 G^{(\pi)}$ & $A_{2}^{(\pi)}=15 A^{(\pi)}$ & $G_{2}^{(\pi)}=G_{2}^{(\nu)}=17 G$ \\
$G^{(\nu)}=0.50$ & $A^{(\nu)}=1.00$ & $G^{(\pi)}=0.50$ & $A^{(\pi)}=1.00$ & $G_{3}^{(\pi)}=G_{3}^{(\nu)}=20 G$ \\
& & & $G=0.7$ \\
\hline
\end{tabular}

As shown in Tables V and VI, besides the ground state, 11 (10) low-lying levels up to that of $2_{5}^{+}$in ${ }^{18} \mathrm{O}\left({ }^{18} \mathrm{Ne}\right)$, together with 10 (4) B(E2) values, are considered to estimate the 4 or 5 adjustable parameters in either the MSP (MSP0) or the NLP (NLP0) after the exact (approximate) projection. It is shown that the pairing interactions within the same Nilsson levels with $G_{3}>G_{2}>G_{1}$ in the MSP (MSP0) or with $A_{3}>A_{2}>A_{1}$ in the NLP (NLP0) can be adjusted not only to produce appropriate energy gaps among these levels, but also to pull the $0_{\eta}^{+}$with $\eta=2,3$ level energies down, otherwise the $0_{\eta}^{+}$with $\eta=2,3$ level energies are much too higher in comparison to the corresponding experimental values. It is clearly shown in Tables $\mathrm{V}$ and VI that both the exact and approximate angular momentum projection provide similar low-lying level patterns for ${ }^{18} \mathrm{O}$ and ${ }^{18} \mathrm{Ne}$, provided that the effective interaction between the valence particles is reasonably adjusted in each case. However, there are still noticeable deviations in higher excited level energies, such as those of $2_{3-5}^{+}$, and that of $4_{2}^{+}$. The deviations are even larger in the exact projection, which implies that the model only describes low-lying spectrum reasonably well. Moreover, the energy ratio $R_{4 / 2} \equiv E_{4_{1}^{+}} / E_{2_{1}^{+}}$ in the exact projection for both ${ }^{18} \mathrm{O}$ and ${ }^{18} \mathrm{Ne}$ is too small in comparison to the corresponding experimental value, while it is improved in the approximate projection. In general, the theoretical energies of higher-lying excited levels are not as well reproduced as the ones of the lower-lying levels. Since the $1_{1}^{+}$level in ${ }^{18} \mathrm{Ne}$ is not determined experimentally, its deviation is not included in the corresponding $\chi$. Both the level energies and the $\mathrm{B}(\mathrm{E} 2)$ values in ${ }^{18} \mathrm{O}$ shown in Tables V and VI, respectively, are reproduced slightly better in the approximate projection than in the exact projection, while the $\mathrm{B}(\mathrm{E} 2)$ values in ${ }^{18} \mathrm{Ne}$ shown in Table VI are reproduced better in the exact projection than those in the approximate projection. Nonetheless, the overall features of the MSP and NLP models in both the exact and approximate projection for these two nuclei are quite the same. Most notable discrepancies of the model in the exact projection are that (1) higher excited level energies are about 4-5 MeV higher than the corresponding experimental data; (2) the ratio $R_{4 / 2}$ is about 1.5 times smaller; and (3) $\mathrm{B}(\mathrm{E} 2)$ values among low-lying states are smaller in comparison to the corresponding experimental values. These discrepancies seem to emerge as a result of the replacement of the quadrupole-quadrupole interaction among like-nucleons by the quadrupole deformation in the deformed mean-field.

TABLE V: Low-lying level energies (in $\mathrm{MeV}$ ) in ${ }^{18} \mathrm{O}$ and ${ }^{18} \mathrm{Ne}$.

\begin{tabular}{|c|c|c|c|c|c|c|c|c|c|c|}
\hline \multirow[b]{2}{*}{$J_{\eta}^{\pi}$} & \multirow[b]{2}{*}{ Exp. [44] } & \multicolumn{4}{|c|}{${ }^{18} \mathrm{O}$} & \multicolumn{5}{|c|}{${ }^{18} \mathrm{Ne}$} \\
\hline & & MSP & MSP0 & NLP & NLP0 & Exp. [44] & MSP & MSP0 & NLP & NLP0 \\
\hline $0_{\mathrm{g}}^{+}$ & 0.0 & 0.0 & 0.0 & 0.0 & 0.0 & 0.0 & 0.0 & 0.0 & 0.0 & 0.0 \\
\hline $0_{2}^{+}$ & 3.633 & 3.74 & 4.93 & 3.13 & 4.06 & 3.576 & 5.12 & 5.94 & 4.17 & 4.17 \\
\hline $0_{3}^{+}$ & 5.336 & 5.89 & 6.70 & 7.32 & 7.27 & 4.590 & 7.29 & 10.61 & 7.01 & 7.01 \\
\hline $2_{1}^{+}$ & 1.982 & 1.91 & 1.31 & 1.73 & 1.09 & 1.887 & 2.56 & 1.02 & 1.97 & 0.78 \\
\hline $2_{2}^{+}$ & 3.920 & 5.98 & 5.71 & 5.35 & 6.04 & 3.616 & 6.31 & 5.32 & 5.68 & 4.88 \\
\hline $2_{3}^{+}$ & 5.255 & 11.15 & 5.78 & 10.51 & 6.13 & 5.090 & 11.52 & 10.96 & 10.89 & 5.64 \\
\hline $2_{4}^{+}$ & 8.213 & 14.58 & 11.14 & 13.95 & 11.48 & 7.059 & 14.59 & 11.79 & 13.99 & 11.00 \\
\hline $2_{5}^{+}$ & 11.390 & 17.06 & 14.39 & 16.32 & 14.73 & 7.910 & 17.50 & 13.70 & 16.94 & 12.70 \\
\hline $4_{1}^{+}$ & 3.555 & 2.53 & 2.67 & 2.03 & 2.91 & 3.376 & 2.96 & 2.55 & 2.41 & 2.50 \\
\hline $4_{2}^{+}$ & 7.117 & 11.15 & 10.88 & 10.51 & 11.22 & 6.297 & 11.55 & 10.35 & 10.92 & 9.55 \\
\hline $3_{1}^{+}$ & 5.378 & 5.99 & 5.79 & 5.35 & 6.13 & 4.561 & 6.39 & 5.61 & 5.76 & 4.82 \\
\hline \multirow[t]{2}{*}{$1_{1}^{+}$} & 8.817 & 11.15 & 10.97 & 10.51 & 11.30 & - & 11.55 & 10.71 & 10.92 & 9.92 \\
\hline & & $\chi=3.51$ & $\chi=2.04$ & $\chi=3.13$ & $\chi=2.26$ & & $\chi=4.88$ & $\chi=3.92$ & $\chi=4.44$ & $\chi=2.43$ \\
\hline
\end{tabular}


TABLE VI: The electric quadrupole moment $Q\left(2_{1}^{+}\right)$(in eb) and B(E2) values (in W.u.) of the transitions between low-lying states in ${ }^{18} \mathrm{O}$ and ${ }^{18} \mathrm{Ne}$, in which "-" denotes that the corresponding value was not determined experimentally. The effective charge is adjusted to be, for ${ }^{18} \mathrm{O}, q_{2}^{(\nu)}=0.60(0.84) e$ in the $\mathrm{MSP}(\mathrm{MSP} 0)$, and $q_{2}^{(\nu)}=1.00(1.00) e$ in the NLP(NLP0), respectively, while for ${ }^{18} \mathrm{Ne}$, to be $q_{2}^{(\pi)}=1.4(1.9) e$ in both the MSP(MSP0) and the NLP(NLP0).

\begin{tabular}{|c|c|c|c|c|c|c|c|c|c|c|}
\hline & \multicolumn{6}{|c|}{${ }^{18} \mathrm{O}$} & \multicolumn{4}{|c|}{${ }^{18} \mathrm{Ne}$} \\
\hline & Exp. [44] & MSP & MSP0 & NLP & NLP0 & Exp. [44] & MSP & MSP0 & NLP & NLP0 \\
\hline $\mathrm{B}\left(\mathrm{E} 2,2_{1}^{+} \rightarrow 0_{\mathrm{g}}^{+}\right)$ & 3.32 & 3.23 & 2.45 & 3.31 & 3.08 & 17.7 & 16.17 & 9.11 & 17.71 & 7.82 \\
\hline $\mathrm{B}\left(\mathrm{E} 2,4_{1}^{+} \rightarrow 2_{1}^{+}\right)$ & 1.19 & 1.68 & 1.36 & 1.67 & 1.51 & 8.9 & 9.16 & 4.58 & 8.97 & 3.08 \\
\hline $\mathrm{B}\left(\mathrm{E} 2,0_{2}^{+} \rightarrow 2_{1}^{+}\right)$ & 17 & 0.34 & 1.36 & 0.0003 & 0.0007 & 5.0 & 9.63 & 11.26 & 0.21 & 0.99 \\
\hline $\mathrm{B}\left(\mathrm{E} 2,2_{2}^{+} \rightarrow 0_{\mathrm{g}}^{+}\right)$ & 1.3 & 1.73 & 0.19 & 1.38 & 0.04 & 0.66 & 10.52 & 12.83 & 8.54 & 12.76 \\
\hline $\mathrm{B}\left(\mathrm{E} 2,2_{3}^{+} \rightarrow 0_{2}^{+}\right)$ & 23 & 0.0002 & 0.02 & 0.61 & 0.04 & - & 0.83 & 3.32 & 2.54 & 14.91 \\
\hline $\mathrm{B}\left(\mathrm{E} 2,2_{3}^{+} \rightarrow 4_{1}^{+}\right)$ & 21 & 1.20 & 2.47 & 1.19 & 2.87 & - & 6.51 & 0.005 & 6.51 & 0.41 \\
\hline $\mathrm{B}\left(\mathrm{E} 2,2_{3}^{+} \rightarrow 0_{\mathrm{g}}^{+}\right)$ & 2.15 & 0.52 & 1.66 & 0.41 & 1.54 & - & 3.52 & 0.51 & 2.38 & 0.22 \\
\hline $\mathrm{B}\left(\mathrm{E} 2,0_{3}^{+} \rightarrow 2_{1}^{+}\right)$ & 2.0 & 0.15 & 0.16 & 0.03 & 0.15 & - & 0.08 & 0.11 & 0.56 & 10.27 \\
\hline $\mathrm{B}\left(\mathrm{E} 2,4_{2}^{+} \rightarrow 2_{2}^{+}\right)$ & 2.2 & 1.45 & 0.42 & 1.45 & 0.78 & - & 7.92 & 5.88 & 7.95 & 7.75 \\
\hline $\mathrm{B}\left(\mathrm{E} 2,2_{4}^{+} \rightarrow 4_{1}^{+}\right)$ & 2.4 & 0.00 & 1.00 & 0.00 & 1.51 & - & 0.0007 & 4.29 & 0.00 & 4.23 \\
\hline & & $\chi=11.01$ & $\chi=10.62$ & $\chi=10.94$ & $\chi=10.74$ & & $\chi=5.50$ & $\chi=8.36$ & $\chi=4.61$ & $\chi=8.57$ \\
\hline$Q\left(2_{1}^{+}\right)$ & $-0.036[45]$ & -0.027 & -0.028 & -0.027 & -0.032 & - & -0.063 & -0.089 & -0.065 & -0.102 \\
\hline
\end{tabular}

Since the approximation projection describes low-lying spectrum of ${ }^{18} \mathrm{O}$ and ${ }^{18} \mathrm{Ne}$ reasonably well, and the fitting qualities of the MSP and NLP models are quite the same, we only use the MSP0 to describe ${ }^{20} \mathrm{Ne}$, namely valence neutrons and protons in the deformed $d s$-shell with the MSP0 will be considered, of which the adjustable MSP parameters used are shown in Table IV. Both proton and neutron sectors of the mean-field plus the modified standard pairing model Hamiltonian are considered, of which the Hamiltonian before the projection is given by

$$
\hat{H}=\hat{H}^{\pi}+\hat{H}^{\nu}
$$

where $\hat{H}^{\pi}$ for the proton sector and $\hat{H}^{\nu}$ for the neutron sector are all taken as the MSP form, while possible residual interactions between valence neutrons and protons are neglected. ${ }^{20} \mathrm{Ne}$ was studied extensively as a typical example within the $d s$-shell in shell model calculations [46-52], and the variation-after-projection Hartree-Fock method [53]. It was confirmed that the wavefunctions of the system are very similar to those given by the Elliott SU(3) model [46, 48, 49]. Most importantly, the coexistence of a shell-model aspect and an $\alpha$-cluster aspect in ${ }^{20} \mathrm{Ne}$ was revealed [50], which can also be equivalently and more rigorously described by the symplectic shell model [41-43] and more recent symplectic no-core shell-model approach [54]. From the analysis in these works, it is shown that the wavefunctions of the proton sector and that of the neutron sector of ${ }^{20} \mathrm{Ne}$ are quite similar, though, in most cases, the eigenstates of ${ }^{20} \mathrm{Ne}$ were provided within the SU(3) basis. In this work, as shown in Table III, the single-particle energies of neutrons and protons generated from the axially deformed Nilsson code are the same. Therefore, it seems reasonable to use the same set of pairing interaction parameters for both the proton sector and the neutron sector in (57). However, the proton-neutron permutation symmetry occurs when the same set of pairing interaction parameters for both the proton sector and neutron sector is adopted. As a result, many level energies in the model become two-fold degenerate due to the proton-neutron permutation symmetry, which must be avoid in order to describe the experimental spectrum of ${ }^{20} \mathrm{Ne}$ in the deformed mean-field plus the modified pairing model. Therefore, we keep the adjustable parameters $G_{i}^{(\pi)}=G_{i}^{(\nu)}$ with $i=2$ and 3 , and take $G_{11}^{(\pi)}$ and $G_{22}^{(\pi)}$ slightly different from $G_{11}^{(\nu)}$ and $G_{22}^{(\nu)}$, respectively, in order to avoid this artificial permutation symmetry, which thus leads to the 7 adjustable parameters used in our calculations shown in Table IV.

As a further approximation, only $K=K^{\pi}+K^{\nu}=0$ intrinsic states are taken in the projection, and hence, possible intrinsic states with total angular momentum quantum number $J$ and $K=0$ may be expressed as

$$
\left|\tau=\left(\tau^{\pi}, J^{\pi}, \tau^{\nu}, J^{\nu}\right), J, K=0\right\rangle=\sum_{\xi_{\pi} \xi_{\nu}} \phi_{\xi_{\pi}}^{\tau J K=0} \xi_{\nu}\left|\xi_{\pi} \xi_{\nu}\right\rangle
$$

where $\left|\xi_{\pi} \xi_{\nu}\right\rangle$ are eigenstates of (57) in the deformed mean-field plus the MSP before the angular momentum projection, and the overlap is given by

$$
\phi_{\xi_{\pi}}^{\tau J K} \xi_{\nu}=0=\sum_{K^{\prime}}\left\langle J^{\pi} K^{\prime}, J^{\nu}-K^{\prime} \mid J 0\right\rangle \phi_{\xi_{\pi}}^{\tau^{\pi} J^{\pi} K^{\prime}} \phi_{\xi_{\nu}}^{\tau^{\nu} J^{\nu}-K^{\prime}}
$$


Since the dimension of the two-particle $d s$-shell subspace is 66 , the dimension of the subspace spanned by two-neutron and two-proton product states is $66^{2}=4356$. However, the dimension of the $K=K^{\pi}+K^{\nu}=0$ subspace is only 640 . Specifically, the number of intrinsic two-neutron and two-proton states with a given $J$ and $K=K^{\pi}+K^{\nu}=0$ are shown in Table VII. Thus, the size of the energy matrix with a given $J$ is greatly reduced. The final results of our model calculations with the single-particle energies given in Table III and the adjustable parameters shown in Table IV are shown in Table VIII, in which the level energies up to $2_{14}^{+}$at $12.221 \mathrm{MeV}$ of ${ }^{20} \mathrm{Ne}$, together with the electric quadrupole moment $Q\left(2_{1}^{+}\right)$(in eb), and B(E2) values (in W.u.) of some transitions between low-lying states in ${ }^{20}$ Ne, are shown.

TABLE VII: The number of intrinsic two-neutron and two-proton states with a given $J$ and $K=K^{\pi}+K^{\nu}=0$ shown in (58).

\begin{tabular}{cccc}
\hline$J$ & The number of $K=0$ states & $J$ & The number of $K=0$ states \\
\hline 0 & 46 & 1 & 101 \\
2 & 143 & 3 & 129 \\
4 & 105 & 5 & 64 \\
6 & 36 & 7 & 12 \\
8 & 4 & & \\
\hline
\end{tabular}

The fitting quality of the lowest 38 level energies of ${ }^{20} \mathrm{Ne}$ seems the same as that of ${ }^{18} \mathrm{O}$ and ${ }^{18} \mathrm{Ne}$ with $\chi=2.35$. In previous studies, generally, the level energies within the yrast band can be well fitted, while the non-yrast states were not described well. It can be observed in Table $\mathrm{V}$ that these level energies are globally well fitted. It can be noticed that the approximate $S_{2}$ symmetry with the permutation of neutrons and protons indeed emerges in level energies predicted in the MSP, which is due to the fact that the single-particle energies of neutrons and protons in the Nilsson model are the same, and the MSP parameters for the proton and neutron sectors are almost the same. Actually, the approximate two-level degeneracy indeed occurs in ${ }^{20} \mathrm{Ne}$. It is shown in Table VIII that the level energies of $0_{5}^{+}$and $0_{6}^{+}, 2_{5}^{+}$and $2_{6}^{+}, 2_{7}^{+}$and $2_{8}^{+}, 2_{9}^{+}$and $2_{10}^{+}, 2_{12}^{+}$and $2_{13}^{+}, 4_{4}^{+}$and $4_{5}^{+}, 4_{6}^{+}$and $4_{7}^{+}, 3_{1}^{+}$and $3_{2}^{+}, 3_{3}^{+}$and $3_{4}^{+}$are indeed almost degenerate. However, there are also obvious contradictions to the approximate $S_{2}$ symmetry in some low-lying level energies. For example, the experimental energy gap of $2_{1}^{+}$and $2_{2}^{+}$is $5.79 \mathrm{MeV}$, and that of $4{ }_{1}^{+}$and $4_{2}^{+}$is $4.78 \mathrm{MeV}$, while the corresponding model predictions are only $0.15 \mathrm{MeV}$, which may be improved by taking isospin-dependent pairing interactions into consideration, though the model augmented by such interactions before the angular momentum projection will no longer be exactly solvable. Similar to the results of ${ }^{18} \mathrm{O}$ and ${ }^{18} \mathrm{Ne}$, larger deviations in energy still occur in some higher excited states. Obvious discrepancies include that the $0_{5}^{+}$and $0_{6}^{+}$level energies are about $4.26 \mathrm{MeV}$ higher than the corresponding experimental values, and that the position of $5_{1}^{+}$state is too low with excitation energy $11.86 \mathrm{MeV}$, which, however, has never been observed in experiment. It can also be observed from Table VIII that the $1_{1}^{+}$level is described better in the MSP0, while it is much too low in energy obtained from the SU(3) shell model $[42,43]$ in comparison to the corresponding experimental value. With the MSP parameters used to calculate these level energies, the electric quadrupole moment $Q\left(2_{1}^{+}\right)$is also well reproduced with $Q\left(2_{1}^{+}\right)=-0.20$ eb in comparison to the corresponding experimental value $Q_{\exp }\left(2_{1}^{+}\right)=-0.23(3)$ eb, while it has been predicted to be around -0.15 eb by previous shell models $[49,50]$, the $\mathrm{SU}(3)$ shell model [41], and the symplectic no-core shell model that uses no effective charges [54]. According to [42], the proton and neutron charges used in the electric quadrupole operator are given as $q_{2}^{\pi}=\left(1+e_{\mathrm{eff}}\right) e$ and $q_{2}^{\nu}=e_{\mathrm{eff}} e$. In adjusting the electric quadrupole moment $Q\left(2_{1}^{+}\right)$and the $\mathrm{B}(\mathrm{E} 2)$ values of ${ }^{20} \mathrm{Ne}, e_{\mathrm{eff}}=1.0$ is taken, which is larger than that used in $[50]$ with $e_{\mathrm{eff}}=0.15$, in $[52,54]$ with $e_{\mathrm{eff}}=0$, and in $[42,43]$ with $e_{\mathrm{eff}}=0.558$. As a result, $\mathrm{B}\left(\mathrm{E} 2,4_{1}^{+} \rightarrow 2_{1}^{+}\right), \mathrm{B}\left(\mathrm{E} 2,6_{1}^{+} \rightarrow 4_{1}^{+}\right)$, and $\mathrm{B}\left(\mathrm{E} 2,8_{1}^{+} \rightarrow 6_{1}^{+}\right)$within the yrast band are smaller than the corresponding experimental values. For the current MSP parameters used, the largest discrepancy occurs in $\mathrm{B}\left(\mathrm{E} 2,0_{3}^{+} \rightarrow 2_{1}^{+}\right)$and $\mathrm{B}\left(\mathrm{E} 2,2_{2}^{+} \rightarrow 2_{1}^{+}\right)$, which are too large in comparison to the corresponding experimental data. Such large B(E2, $\left.0_{3}^{+} \rightarrow 2_{1}^{+}\right)$and B(E2, $\left.2_{2}^{+} \rightarrow 2_{1}^{+}\right)$values indicates a similar largely deformed prolate structure of the valence particles for both $0_{3}^{+}, 2_{2}^{+}$, and $2_{1}^{+}$states. However, as the $2_{2}^{+}$and $0_{3}^{+}$states are calculated to lie between the ${ }^{16} \mathrm{O}+\alpha($ at $4.73 \mathrm{MeV})$ and ${ }^{12} \mathrm{C}+2 \alpha($ at $11.89 \mathrm{MeV})$ thresholds, core excitations, if augmented in the model, may form an oblate substructure of ${ }^{12} \mathrm{C}$ and change the overall $2_{2}^{+}$and $0_{3}^{+}$structure, thereby considerably decreasing the $\mathrm{B}\left(\mathrm{E} 2,0_{3}^{+} \rightarrow 2_{1}^{+}\right)$and $\mathrm{B}\left(\mathrm{E} 2,2_{2}^{+} \rightarrow 2_{1}^{+}\right)$values.

While low-lying spectra can be described fairly well, to study whether the strong pairing interactions within the same Nilsson levels used are reasonable, we have calculated the pairing contribution to the binding energies relative to the ${ }^{16} \mathrm{O}$ core in these nuclei, because the valence particles in these nuclei are restricted within the $d s$-shell. We do not intend to provide the binding energies of these nuclei from the present model, but using the pairing interaction parameters shown in Table IV, we find that the pairing contribution to the relative binding energy is reasonable, namely, it is less than $40 \%$ in ${ }^{18} \mathrm{Ne}$, less than $18.5 \%$ in ${ }^{20} \mathrm{Ne}$, and less than $20 \%$ in ${ }^{18} \mathrm{O}$ (Table IX). Though the pairing contribution to the relative binding energy in ${ }^{18} \mathrm{Ne}$ seems large, the total contribution per particle is small 
TABLE VIII: Low-lying level energies (in MeV), the electric quadrupole moment $Q\left(2_{1}^{+}\right)$(in eb), and B(E2) values (in W.u.) of some transitions between low-lying states in ${ }^{20} \mathrm{Ne}$ calculated in the approximate projection scheme, in which "-" denotes that the corresponding level was not observed experimentally. The effective charges used in the calculations are $q_{2}^{(\pi)}=2.0 e$ and $q_{2}^{(\nu)}=1.0 e$.

\begin{tabular}{|c|c|c|c|c|c|c|c|c|c|}
\hline$J_{\eta}^{\pi}$ & Exp. [55] & MSP0 & $J_{\eta}^{\pi}$ & Exp. [55] & MSP0 & & Exp. [55] & MSP0 & Other work \\
\hline $0_{\mathrm{g}}^{+}$ & 0.0 & 0.0 & $4_{1}^{+}$ & 4.248 & 3.52 & $\mathrm{~B}\left(\mathrm{E} 2,2_{1}^{+} \rightarrow 0_{\mathrm{g}}^{+}\right)$ & $20.3(10)$ & 13.77 & $17.67[50], 21.80[52]$ \\
\hline $0_{2}^{+}$ & 6.725 & 8.16 & $4_{2}^{+}$ & 9.031 & 3.67 & & & & $16.56[42], 14.91[43], 18.5[54]$ \\
\hline $0_{3}^{+}$ & 7.191 & 8.24 & $4_{3}^{+}$ & 9.990 & 8.29 & $\mathrm{~B}\left(\mathrm{E} 2,4_{1}^{+} \rightarrow 2_{1}^{+}\right)$ & $22(2)$ & 4.11 & $21.67[50], 25.95[52]$ \\
\hline $0_{4}^{+}$ & 8.700 & 12.81 & $4_{4}^{+}$ & 10.553 & 10.05 & & & & $21.71[42], 18.30[43], 24.6[54]$ \\
\hline $0_{5}^{+}$ & 10.970 & 15.16 & $4_{5}^{+}$ & 10.800 & 10.13 & $\mathrm{~B}\left(\mathrm{E} 2,0_{2}^{+} \rightarrow 2_{1}^{+}\right)$ & 3.6 & 3.16 & $1.23[50], 4.25[42], 0.217[43]$ \\
\hline $0_{6}^{+}$ & 11.558 & 15.89 & $4_{6}^{+}$ & 11.020 & 10.18 & $\mathrm{~B}\left(\mathrm{E} 2,0_{3}^{+} \rightarrow 2_{1}^{+}\right)$ & $0.31(6)$ & 52.68 & $0.10[42], 0.772[43]$ \\
\hline $2_{1}^{+}$ & 1.634 & 0.68 & $4_{7}^{+}$ & 11.090 & 10.37 & $\mathrm{~B}\left(\mathrm{E} 2,2_{2}^{+} \rightarrow 2_{1}^{+}\right)$ & $1.7(2)$ & 63.33 & $0.38[50]$ \\
\hline $2_{2}^{+}$ & 7.422 & 0.83 & $4_{8}^{+}$ & 11.928 & 11.75 & $\mathrm{~B}\left(\mathrm{E} 2,2_{3}^{+} \rightarrow 0_{\mathrm{g}}^{+}\right)$ & $0.73(9)$ & 2.29 & $0.09[49]$ \\
\hline $2_{3}^{+}$ & 7.833 & 5.56 & $6_{1}^{+}$ & 8.778 & 9.81 & $\mathrm{~B}\left(\mathrm{E} 2,4_{2}^{+} \rightarrow 2_{1}^{+}\right)$ & $5.8(7)$ & 29.77 & $0.28[50]$ \\
\hline $2_{4}^{+}$ & 9.000 & 5.64 & $6_{2}^{+}$ & 12.137 & 9.90 & $\mathrm{~B}\left(\mathrm{E} 2,4_{3}^{+} \rightarrow 2_{1}^{+}\right)$ & $8.3(37)$ & 0.87 & $0.22[50]$ \\
\hline $2_{5}^{+}$ & 9.196 & 8.27 & $8_{1}^{+}$ & 11.951 & 10.96 & $\mathrm{~B}\left(\mathrm{E} 2,2_{7}^{+} \rightarrow 0_{\mathrm{g}}^{+}\right)$ & $0.095(26)$ & 12.56 & $0.85[42], 0.043[43]$ \\
\hline $2_{6}^{+}$ & 9.487 & 8.41 & $3_{1}^{+}$ & 9.873 & 7.75 & $\mathrm{~B}\left(\mathrm{E} 2,6_{1}^{+} \rightarrow 4_{1}^{+}\right)$ & $20(3)$ & 0.001 & $17.08[50], 16.34[52]$, \\
\hline $2_{7}^{+}$ & 10.273 & 8.79 & $3_{2}^{+}$ & 10.884 & 7.84 & & & & $14.31[43], 23.0[54]$ \\
\hline $2_{8}^{+}$ & 10.584 & 8.91 & $3_{3}^{+}$ & 10.917 & 11.45 & $\mathrm{~B}\left(\mathrm{E} 2,8_{1}^{+} \rightarrow 6_{1}^{+}\right)$ & 7.44 & 2.68 & $8.81[50], 6.51[52], 8.99[43]$ \\
\hline $2_{9}^{+}$ & 10.843 & 10.00 & $3_{4}^{+}$ & 11.555 & 11.85 & & & & \\
\hline $2_{10}^{+}$ & 10.940 & 10.63 & $3_{5}^{+}$ & 11.653 & 14.09 & & & $\chi=27.16$ & \\
\hline $2_{11}^{+}$ & 11.116 & 10.98 & $1_{1}^{+}$ & 9.935 & 10.95 & & & & \\
\hline $2_{12}^{+}$ & 11.320 & 13.06 & $1_{2}^{+}$ & 11.262 & 11.73 & $Q\left(2_{1}^{+}\right)$ & $-0.23(3)[56]$ & -0.20 & $-0.152[50],-0.15[41],-0.157[54]$ \\
\hline $2_{13}^{+}$ & 11.885 & 13.67 & $5_{1}^{+}$ & - & 11.86 & & & & \\
\hline $2_{14}^{+}$ & 12.221 & 14.05 & & & $\chi=2.35$ & & & & \\
\hline
\end{tabular}

with $\bar{E}_{\mathrm{P}} \leq 0.882 \mathrm{MeV}$, which seems reasonable in comparison with the pairing energy per particle in the adjacent nuclei shown in Table IX. As is well known, the pairing interaction strength in the standard pairing model decreases with the increasing of the number of valence particles. It is expected that these 'on-site' pairing interactions will become weaker for a larger number of valence particles.

TABLE IX: Pairing energy per valence particle $\bar{E}_{P}=\left|E_{P}\right| / n$ (in MeV), pairing energy contribution to the relative binding energy in the MSP0 and NLP0 for ${ }^{18} \mathrm{O},{ }^{18} \mathrm{Ne}$, and ${ }^{20} \mathrm{Ne}$, respectively, quantified by the ratio $R=E_{\mathrm{P}} / E_{R B}$, in which $E_{\mathrm{P}}=$ $\left\langle 0_{\mathrm{g}}^{+}\left|\tilde{H}_{\mathrm{P}}\right| 0_{\mathrm{g}}^{+}\right\rangle$(in $\mathrm{MeV}$ ), where $\tilde{H}_{\mathrm{P}}$ is the pairing interaction part of (48) with all single-particle energies zero and the pairing interaction parameters shown in Table IV, and $E_{R B}=E_{B}-E_{B}\left({ }^{16} O\right)$, in which $E_{B}$ is taken from [57].

\begin{tabular}{rccccc}
\hline & \multicolumn{2}{c}{${ }^{18} \mathrm{O}$} & \multicolumn{2}{c}{${ }^{18} \mathrm{Ne}$} & ${ }^{20} \mathrm{Ne}$ \\
& $\mathrm{MSP}$ & $\mathrm{NLP}$ & $\mathrm{MSP}$ & $\mathrm{NLP}$ & $\mathrm{MSP}$ \\
\hline $\bar{E}_{\mathrm{P}}$ & 1.185 & 1.184 & 0.882 & 0.752 & 1.566 \\
$R$ & $19.4 \%$ & $19.4 \%$ & $39.0 \%$ & $33.2 \%$ & $18.5 \%$ \\
\hline
\end{tabular}

Finally, in order to show the applicability of the model with larger configuration subspace with less free parameters, ${ }^{24} \mathrm{Mg}$ is chosen to be fitted by the NLP with approximate angular momentum projection, which is described by the model with 4 neutrons and 4 protons in the $d s$ shell similar to the ${ }^{20} \mathrm{Ne}$ case. The dimension of the subspace is 245,025 , while there are only 28,503 intrinsic states with $K=0$ considered in the approximate angular momentum projection. Besides the single-particle energies generated from the Nilsson model, the parameter $B=0.06 \mathrm{MeV}^{-2}$ is used with the pairing interaction strengthes $A_{1}=0.5 A, A_{2}=3.5 A$, and $A_{3}=4.5 A$ for both the proton and neutron sectors, where $A=3.7 \mathrm{MeV}$, while $A_{22}^{\nu}=0.5 A$ and $A_{22}^{\pi}=1.5 A$, are used, similar to the ${ }^{20} \mathrm{Ne}$ case, in order to avoid the proton-neutron permutation symmetry. The results together with $\mathrm{B}(\mathrm{E} 2)$ values obtained from the $a b$ initio symmetry-adapted no-core shell model (SA-NCSM), which were not published previously, and those of the SU(3) shell model and the $\operatorname{Sp}(4, \mathrm{R})$ model [58] are shown in Table X. The fitting quality of level energies of ${ }^{24} \mathrm{Mg}$ with $\chi=3.12$ is quite similar to that of ${ }^{20} \mathrm{Ne}$. As shown in Table X, though the 20 excited level energies are globally fitted reasonably 
well, deviations in higher lying level energies are obvious. Generally speaking, the E2 transition amplitudes produced from the model is smaller in comparison to the experimental data and those of the SA-NCSM. Therefore, larger effective charges should be used. In addition, $\mathrm{B}\left(\mathrm{E} 2,3_{1}^{+} \rightarrow 2_{1}^{+}\right)$obtained from the model is too small, while $\mathrm{B}(\mathrm{E} 2$, $2_{2}^{+} \rightarrow 0_{\mathrm{g}}^{+}$) is too large in comparison to the corresponding experimental data. It is expected that these discrepancies may be overcome by taking isospin-dependent pairing interactions and other configuration mixing calculations into consideration.

TABLE X: Low-lying level energies (in MeV), the electric quadrupole moment $Q\left(2_{1}^{+}\right.$) (in eb), and B(E2) values (in W.u.) of some transitions between low-lying states in ${ }^{24} \mathrm{Mg}$ calculated in the NLP with the approximate projection scheme, in which "-" denotes that the corresponding $\mathrm{B}(\mathrm{E} 2)$ value was not observed experimentally. The effective charges used in the calculations are $q_{2}^{(\pi)}=2.0 e$ and $q_{2}^{(\nu)}=1.0 e$. The results with $(*)$ are obtained from the SA-NCSM.

\begin{tabular}{|c|c|c|c|c|c|c|c|c|c|c|c|}
\hline$J_{\eta}^{\pi}$ & Exp. [59] & NLP0 & $J_{\eta}^{\pi}$ & Exp. [59] & NLP0 & & Exp. [59] & NLP0 & \multicolumn{3}{|c|}{ Other work } \\
\hline $0_{1}^{+}$ & 0.0 & 0.0 & $4_{1}^{+}$ & 4.123 & 2.35 & $\mathrm{~B}\left(\mathrm{E} 2,2_{1}^{+} \rightarrow 0_{\mathrm{g}}^{+}\right)$ & $21.50(10)$ & 16.72 & $29.00^{(*)}$ & $6.90[58]$ & $26.30[58]$ \\
\hline $0_{2}^{+}$ & 6.432 & 6.80 & $4_{2}^{+}$ & 6.011 & 2.63 & $\mathrm{~B}\left(\mathrm{E} 2,4_{1}^{+} \rightarrow 2_{1}^{+}\right)$ & $39.00(4)$ & 25.92 & $33.00^{(*)}$ & $9.50[58]$ & $36.50[58]$ \\
\hline $0_{3}^{+}$ & 9.305 & 7.18 & $4_{3}^{+}$ & 8.439 & 7.74 & $\mathrm{~B}\left(\mathrm{E} 2,6_{1}^{+} \rightarrow 4_{1}^{+}\right)$ & - & 7.51 & $32.00^{(*)}$ & $9.70[58]$ & $39.30[58]$ \\
\hline $2_{1}^{+}$ & 1.369 & 1.85 & $4_{4}^{+}$ & 9.301 & 8.49 & $\mathrm{~B}\left(\mathrm{E} 2,2_{2}^{+} \rightarrow 0_{\mathrm{g}}^{+}\right)$ & $1.94(19)$ & 20.40 & $4.40^{(*)}$ & $2.50[58]$ & $4.60[58]$ \\
\hline $2_{2}^{+}$ & 4.238 & 2.34 & $4_{5}^{+}$ & 9.516 & 8.60 & $\mathrm{~B}\left(\mathrm{E} 2,3_{1}^{+} \rightarrow 2_{1}^{+}\right)$ & $58.00(8)$ & 0.00 & $53.00^{(*)}$ & & \\
\hline $2_{3}^{+}$ & 7.349 & 3.20 & $4_{6}^{+}$ & 9.532 & 8.80 & $\mathrm{~B}\left(\mathrm{E} 2,3_{1}^{+} \rightarrow 2_{2}^{+}\right)$ & $2.50(3)$ & 4.16 & $7.80^{(*)}$ & & \\
\hline $2_{4}^{+}$ & 8.655 & 3.85 & $6_{1}^{+}$ & 7.114 & 8.37 & $\mathrm{~B}\left(\mathrm{E} 2,2_{2}^{+} \rightarrow 2_{1}^{+}\right)$ & $3.60(4)$ & 1.62 & & & \\
\hline $2_{5}^{+}$ & 9.003 & 4.30 & $6_{2}^{+}$ & 9.528 & 9.07 & $\mathrm{~B}\left(\mathrm{E} 2,0_{2}^{+} \rightarrow 2_{2}^{+}\right)$ & $8.90(14)$ & 0.01 & & & \\
\hline $2_{6}^{+}$ & 9.248 & 5.38 & $3_{1}^{+}$ & 5.235 & 11.00 & $\mathrm{~B}\left(\mathrm{E} 2,0_{2}^{+} \rightarrow 2_{1}^{+}\right)$ & $0.64(10)$ & 0.43 & & & \\
\hline $1_{1}^{+}$ & 9.828 & 15.98 & $3_{2}^{+}$ & 9.457 & 11.55 & $\mathrm{~B}\left(\mathrm{E} 2,2_{3}^{+} \rightarrow 0_{\mathrm{g}}^{+}\right)$ & $0.67(23)$ & 0.89 & & & \\
\hline $5_{1}^{+}$ & 7.812 & 11.64 & & & $\chi=3.12$ & & & $\chi=21.04$ & & & \\
\hline & & & & & & $Q\left(2_{1}^{+}\right)$ & $-0.166(6)$ & -0.22 & $-0.22^{(*)}$ & & \\
\hline
\end{tabular}

\section{SUMMARY}

The angular momentum projection for the axially deformed Nilsson mean-field plus a modified standard pairing (MSP) or the nearest-level pairing (NLP) model is proposed. Exact solutions of the MSP are formulated by using the Richardson-Gaudin method, of which the solutions can be obtained by using the polynomial approach shown in $[28,29]$. Both the exact projection, in which all intrinsic states are taken in the projection, and the approximate projection, in which only intrinsic states with $K=0$ are taken in the projection, are considered. Our analysis shows that the approximate projection with $K=0$ intrinsic states seems a reasonable approximation, especially for low-lying states. With the approximation, the configuration subspace considered is greatly reduced, especially when the number of valence particles and number of Nilsson levels considered become large. It should be stated that the projection carried out in this work is for a deformed Hamiltonian, in which all intrinsic states should be considered in contrast to the previous angular momentum projection method. The approximate projection is achieved by only taking $K=0$ intrinsic states because $K \neq 0$ states lie higher in energy. Moreover, even in the present approximate projection, all $K=0$ intrinsic states are included instead of only a few low-lying HF (intrinsic) states are taken into consideration in previous angular momentum projection method for a spherical shell model Hamiltonian [25, 36]. The advantage of the method used in $[25,36]$ lies in the fact that the configuration subspace is greatly reduced based on the HF or deformed shell model solutions, which provides us a way to further reduce the dimension of the configuration subspace when more particles in a larger major shell is considered.

As a starting point of the application, low-lying spectra and E2 properties of ${ }^{18} \mathrm{O}$ and ${ }^{18} \mathrm{Ne}$ are calculated by using the exact and approximate angular momentum projection of the axially deformed Nilsson mean-field plus the pairing models, while only the approximate angular momentum projection is carried out for ${ }^{20} \mathrm{Ne}$ and ${ }^{24} \mathrm{Mg}$. It is found that there are obvious discrepancies in some level energies and B(E2) values obtained from the models with angular momentum projection, which are understandable because the quadrupole-quadrupole interaction among likenucleons is approximated as the quadrupole deformation in the deformed mean-field with only pairing interactions among like-nucleon pairs. It is observed that strong pairing interactions among valence nucleon pairs within the same Nilsson levels are needed in both the MSP and the NLP in order to reproduce the non-degenerate low-lying levels of these nuclei. Nevertheless, the strong pairing interactions among valence nucleon pairs within the same Nilsson levels adopted seem still acceptable as far as the pairing contribution to the total binding energy of these nuclei 
are concerned. As shown in the fitting to the level energies and $\mathrm{B}(\mathrm{E} 2)$ values of ${ }^{24} \mathrm{Mg}$, there are only a few $\mathrm{MeV}$ differences among the 'on-site' pairing interaction strengthes. It is expected that the differences among these 'on-site' pairing interaction strengthes will become negligible when the number of valence particles is getting further large. The approximate projection procedure makes it possible to study low-lying spectra of deformed nuclei in other mass regions with more number of valence particles described by a deformed mean-field plus residual interactions, which will be part of our future work. It should be pointed out that the angular momentum projection of deformed meanfield plus pairing models only provides a way to get approximate results of the deformed models with good angular momentum, which may be used to describe deformed nuclei approximately, especially for their low-lying states.

Support from the U. S. National Science Foundation (OCI-0904874, ACI -1516338), U. S. Department of Energy (DE-SC0005248), the Southeastern Universities Research Association, the China-U. S. Theory Institute for Physics with Exotic Nuclei (CUSTIPEN) (DE-FG02-13ER42025), the National Natural Science Foundation of China (11175078, 11375080, and 11465001), and the LSU-LNNU joint research program (9961) is acknowledged.

\section{Appendix A: Parameters used in the Nilsson shell model}

The Hamiltonian of a single nucleon in the Nilsson model used is of the standard form:

$$
\hat{h}=\hat{h}_{\mathrm{HO}}+\hat{h}_{\epsilon_{2}}-\kappa \hbar \omega_{0}\left[2 \hat{l} \cdot \hat{s}+\mu\left(\hat{l}^{2}-\left\langle\hat{l}^{2}\right\rangle_{N_{\mathrm{HO}}}\right]\right)
$$

where $\hat{h}_{\mathrm{HO}}$ is the Hamiltonian of a single nucleon in the spherical harmonic potential, $\kappa$ and $\mu$ are dimensionless parameters, of which the values as suggested by Bengsson and Ragnarsson [60] are shown in Table 1, the average value of $\hat{l}^{2}$ over each major shell, $\left\langle\hat{l}^{2}\right\rangle_{N_{\mathrm{HO}}}$, is given by

$$
\left\langle\hat{l}^{2}\right\rangle_{N_{\mathrm{HO}}}=\frac{1}{2} N_{\mathrm{HO}}\left(N_{\mathrm{HO}}+3\right)
$$

in which $N_{\mathrm{HO}}$ is the principal quantum number of the major shell, and

$$
\hat{h}_{\epsilon_{2}}=-\frac{M_{n}}{2} \omega_{0}^{2} r^{2} \epsilon_{2} \frac{4}{3} \sqrt{\frac{4 \pi}{5}} Y_{20}(\theta, \varphi),
$$

in which $M_{n}$ is the nucleon mass, while $\epsilon_{2}$ is the quadrupole deformation parameter. In our Nilsson shell model code, the Coulomb and Pauli principle effects were incorporated into the potential by choosing

$$
\hbar \omega_{0}=41 A^{-1 / 3}\left(1 \pm \frac{1}{3} \frac{A-2 Z}{A}\right) \mathrm{MeV}
$$

with + sign for neutron and - sign for proton.

TABLE XI: Values of $\kappa$ and $\mu$ used in (A1), which are taken from [60].

\begin{tabular}{lcccc}
\hline & \multicolumn{2}{c}{ Protons } & \multicolumn{2}{c}{ Neutrons } \\
$N_{\text {HO }}$ & $\kappa$ & $\mu$ & $\kappa$ & $\mu$ \\
\hline 0 & 0.120 & 0.00 & 0.120 & 0.00 \\
1 & 0.120 & 0.00 & 0.120 & 0.00 \\
2 & 0.105 & 0.00 & 0.105 & 0.00 \\
3 & 0.090 & 0.30 & 0.090 & 0.25 \\
4 & 0.065 & 0.57 & 0.070 & 0.39 \\
5 & 0.060 & 0.65 & 0.062 & 0.43 \\
6 & 0.054 & 0.69 & 0.062 & 0.34 \\
7 & 0.054 & 0.69 & 0.062 & 0.26 \\
$8 \ldots$ & 0.054 & 0.60 & 0.062 & 0.26 \\
\hline \hline
\end{tabular}




\section{Appendix B: Matrix elements of irreducible tensor operators}

Let $T_{\mu}^{k}$ be rank- $k$ irreducible tensor operators of $R_{3}$ in the laboratory frame. According to (40), we have

$$
T_{\mu}^{k}=\rho_{k} \sum_{\nu} D_{\mu \nu}^{k *}(\alpha, \beta, \gamma) T_{\nu}^{k}(\mathrm{in})
$$

where the scalar factor $\rho_{k}$ should be fixed to ensure the $R_{3}$-reduced matrix elements of $T_{\mu}^{k}$ in the laboratory frame are the same as the corresponding ones in the intrinsic frame.

In the exact angular momentum projection, the eigenstates in the laboratory frame can be expressed in terms of those in the intrinsic frame as

$$
|\eta J M\rangle_{\mathrm{L}}=\sqrt{\frac{1}{8 \pi^{2}}} \sum_{K} D_{M K}^{J *}(\alpha, \beta, \gamma)|\eta J K\rangle .
$$

Therefore, reduced matrix elements of $T_{\mu}^{k}$ in the laboratory frame can be expressed as

$$
{ }_{\mathrm{L}}\left\langle\eta^{\prime} J^{\prime} \| T^{k}|| \eta J\right\rangle_{\mathrm{L}}=\frac{\rho_{k}}{2 J^{\prime}+1} \sum_{K K^{\prime} \nu}\left\langle J K, k \nu \mid J^{\prime} K^{\prime}\right\rangle^{2}\left\langle\eta^{\prime} J^{\prime} \| T^{k}(\text { in }) \| \eta J\right\rangle,
$$

which implies that every component $T_{\nu}^{k}$ (in) is nonzero, where the $R_{3}$ reduced matrix element is defined in terms of CG coefficient of $R_{3}$ by

$$
\left\langle\eta^{\prime} J^{\prime} M^{\prime}\left|T_{q}^{k}\right| \eta J M\right\rangle=\left\langle J M, k q \mid J^{\prime} M^{\prime}\right\rangle\left\langle\eta^{\prime} J^{\prime}|| T^{k} \| \eta J\right\rangle .
$$

Hence, $\left\langle\eta^{\prime} J^{\prime}|| \hat{I}|| \eta J\right\rangle=\delta_{\eta^{\prime} \eta} \delta_{J^{\prime} J}$, where $\hat{I}$ is the unit scalar operator. Therefore, the reduced matrix element ${ }_{\mathrm{L}}\left\langle\eta^{\prime} J^{\prime}\left\|T^{k}\right\| \eta J\right\rangle_{\mathrm{L}}$ exactly equals to $\left\langle\eta^{\prime} J^{\prime} \| T^{k}(\right.$ in) $\| \eta J\rangle$ when $\rho_{k}=1$, which is well-known.

If only $T_{0}^{k}(\mathrm{in})$ is nonzero like the intrinsic quadrupole tensor operator in the Nilsson model, Eq. (B3) should be rewritten as

$$
{ }_{\mathrm{L}}\left\langle\eta^{\prime} J^{\prime}|| T^{k} \| \eta J\right\rangle_{\mathrm{L}}=\frac{\rho_{k}}{2 J^{\prime}+1} \sum_{K}\left\langle J K, k 0 \mid J^{\prime} K\right\rangle^{2}\left\langle\eta^{\prime} J^{\prime} \| T^{k}(\text { in }) \| \eta J\right\rangle .
$$

Since

$$
\sum_{K}\left\langle J K, k 0 \mid J^{\prime} K\right\rangle^{2}=\frac{2 J^{\prime}+1}{2 k+1}
$$

as long as the $R_{3}$ coupling $J \otimes k$ contains $J^{\prime}$, the reduced matrix element ${ }_{\mathrm{L}}\left\langle\eta^{\prime} J^{\prime}\left\|T_{\mu}^{k}\right\| \eta J\right\rangle_{\mathrm{L}}$ exactly equals to $\left\langle\eta^{\prime} J^{\prime}|| T^{k}\right.$ (in) ||$\left.\eta \mathrm{J}\right\rangle$ when and only when $\rho_{k}=2 k+1$ is set. Thus, $\rho_{2}=5$ is used in the exact angular momentum projection for quadrupole tensor operators.

In the approximate angular momentum projection, as shown in (50), the eigenstates in the laboratory frame expressed in terms of those in the intrinsic frame are

$$
|\eta J M\rangle_{\mathrm{L}}=\sqrt{\frac{2 J+1}{8 \pi^{2}}} D_{M 0}^{J *}(\alpha, \beta, \gamma)|\eta J 0\rangle .
$$

Hence, the reduced matrix elements of $T_{\mu}^{k}$ in the laboratory frame can be expressed as

$$
{ }_{\mathrm{L}}\left\langle\eta^{\prime} J^{\prime}|| T^{k} \| \eta J\right\rangle_{\mathrm{L}}=\rho_{k} \sqrt{\frac{2 J+1}{2 J^{\prime}+1}}\left\langle J \quad 0, k 0 \mid J^{\prime} 0\right\rangle^{2}\left\langle\eta^{\prime} J^{\prime} \| T^{k}(\text { in) }) \| \eta J\right\rangle .
$$


In this case, in order to ensure that the reduced matrix elements in the laboratory frame are the same as the corresponding ones in the intrinsic frame, the scalar factor $\rho_{k}$ should also depend on $J$ and $J^{\prime}$. The value of $\rho_{2}$ changes from 2.236 to 2.666 for $J \rightleftharpoons J+2$ transitions with $J \geq 0$, in which $\rho_{2}=2.666$ is the largest value in the large $J$ limit, while $\rho_{2}=2.5$ to 4.0 for $J \rightleftharpoons J$ transitions with $J>1$, in which $\rho_{2}=4.0$ is the largest value in the large $J$ limit. Since the projection is approximate, we uniformly set $\rho_{2}=3.0$ in the approximate angular momentum projection cases in this work.

[1] A. de-Shalit and I. Talmi, Nuclear Shell Theory (Academic Press, New York, 1963)

[2] P. Ring and P. Schuck, The Nuclear Many-Body Problem (Springer-Verlag, Berlin, 1980).

[3] A. Bohr, B. R. Mottelson, and D. Pines, Phys. Rev. 110, 936 (1958).

[4] S. T. Belyaev, Mat. Fys. Medd. K. Dan. Vidensk. Selsk. 31, 11 (1959).

[5] J. P. Elliott, Proc. R. Soc. London A 245, 562 (1958); 272, 557 (1963).

[6] J. P. Elliott, M. Harvey, Proc. Roy. Soc. A 272, 557 (1963).

[7] R. D. Ratna Raju, J. P. Draayer, and K. T. Hecht, Nucl. Phys. A 202, 433 (1973).

[8] G. Rosensteel and D. J. Rowe, Phys. Rev. Lett. 38, 10 (1977).

[9] G. F. Filippov, V. S. Vasilevsky, and L. L. Chopovsky, Sov. J. Part. Nucl. 15, 600 (1984).

[10] D. J. Rowe, Rep. Prog. Phys. 48, 1419 (1985).

[11] D. Troltenier, J. P. Draayer, P. O. Hess, O. Castaños, Nucl. Phys. A 576, 351 (1994).

[12] A. C. Dreyfuss, K. D. Launey, T. Dytrych, J. P. Draayer, and C. Bahri, Phys. Lett. B 727, 511 (2013).

[13] T. Dytrych, K. D. Launey, J. P. Draayer, P. Maris, J. P. Vary, E. Saule, U. Çatalyürek, M. Sosonkina, D. Langr, and M. A. Caprio, Phys. Rev. Lett. 111, 252501 (2013).

[14] J. Dechargé, D. Gogny, Phys. Rev. C, 21, 1568 (1980).

[15] J. Dobaczewski, H. Flocard, and J. Treiner, Nucl. Phys. A 422, 103(1984).

[16] S. T. Belyaev, A. V. Smirnov, S. V. Tolokonnikov, and S. A. Fayans, Sov. J. Nucl. Phys. 45, 783 (1987).

[17] P. Ring, Prog. Part. Nucl. Phys. 37, 193 (1996).

[18] D. Vretenar, A. V. Afanasjev, G. A. Lalazissis, P. Ring, Phys. Rep. 409, 101 (2005).

[19] J. Meng, H. Toki, S. G. Zhou, S. Q. Zhang, W. H. Long, L. S. Geng, Prog. Part. Nucl. Phys. 57, 470 (2006).

[20] M. Bender, P. H. Heenen, and P. G. Reinhard, Rev. Mod. Phys. 75, 121 (2003).

[21] S. G. Nilsson, Dan. Mat. Fys. Medd. 29, 16 (1955).

[22] S. G. Nilsson, C. F. Tsang, A. Sobiczewski, Z. Szymanski, S. Wycech, C. Gustafson, I.-L. Lamm, P. Möler, B. Nilsson, Nucl. Phys. A 131, 1 (1969).

[23] K. W. Schmid, F. Grümmer, and A. Faessler, Phys. Rev. C 29, 291 (1984).

[24] K. W. Schmid, F. Grümmer, and A. Faessler, Phys. Rev. C 29, 308 (1984).

[25] K. Hara and Y. Sun, Int. J. Mod. Phys. E 4, 637 (1995).

[26] R. W. Richardson, Phys. Lett. 3, 277 (1963); 5, 82 (1963); R. W. Richardson and N. Sherman, Nucl. Phys. 52, 221 (1964); 52, 253 (1964).

[27] M. Gaudin, J. Physique 37, 1087 (1976).

[28] X. Guan, K. D. Launey, M. Xie, L. Bao, F. Pan, and J. P. Draayer, Phys. Rev. C 86, 024313 (2012).

[29] X. Guan, K. D. Launey, M. Xie, L. Bao, F. Pan, and J. P. Draayer, Comp. Phys. Commun. 1852714 (2014).

[30] H. Molique and J. Dudek, Phys. Rev. C 56, 1795(1997).

[31] H. C. Pradhan, Y. Nogami, and J. Law, Nucl. Phys. A 201, 357(1973).

[32] H. J. Mang, Phys. Rep. 18, 325 (1975).

[33] F. Pan and J. P. Draayer, J. Phys. A: Math. Gen. 33, 9095(2000).

[34] Y. Chen, F. Pan, G. S. Stoitcheva, and J. P. Draayer, Int. J. Mod. Phys. B 16, 2071 (2002).

[35] F. Pan, Y. Wang, X. Guan, Lu Jia, X.-R. Chen, and J. P. Draayer, AIP Conf. Proc. 1342, 63 (2011).

[36] H. A. Lamme and E. Boeker, Nucl. Phys. A 111, 492 (1968).

[37] F. Pan, B. Li, Y.-Z. Zhang, and J. P. Draayer, Phys. Rev. C 88, 034305 (2013).

[38] D. A. Varshalovich, A. N. Moskalev, and V. K. Khersonskii, Quantum Theory of Angular Momentum (World Scientific, Singapore, 1988).

[39] P. Möller, J. R. Nix, W. D. Myers, and W. J. Swiatecki, Atomic Data Nucl. Data Tables 59, 185 (1995).

[40] G. Audi, O. Bersillon, J. Blachot, A. H. Wapstra, Nucl. Phys. A 624, 1 (1997).

[41] J. P. Draayer and K. J. Weeks, Nucl. Phys. A 413, 215 (1984).

[42] D. Troltenier J. P. Draayer, J. G. Hirsch, Nucl. Phys. A 601, 89 (1996).

[43] C. E. Vargas, J. G. Hirsch, and J. P. Draayer, Nucl. Phys. A 690, 409 (2001).

[44] D. R. Tilley, H. R. Weller, C. M. Cheves, R. M. Chasteler, Nucl. Phys. A 595, 1 (1995).

[45] E. E. Gross and M. P. Fewell, Nucl. Phys. A 411, 329 (1983).

[46] T. Inoue, T. Sebe, H. Hagiwara, and A. Arima, Nucl. Phys. 59, 1 (1964).

[47] T. Inoue, T. Sebe, H. Hagiwara, and A. Arima, Nucl. Phys. 85, 184 (1966).

[48] Y. Akiyama, A. Arima, T. Sebe, Nucl. Phys. A 138, 273 (1969).

[49] A. Arima, M. Sakakura, and T. Sebe, Nucl. Phys. A 170, 273 (1971). 
[50] T. Tomoda and A. Arima, Nucl. Phys. A 303, 217 (1978).

[51] B. A. Brown, B. H. Wildenthal, W. Chung, S. E. Massen, M. Bernas, A. M. Bernstein and R. Miskimen, V. R. Brown and V. A. Madsen, Phys. Rev. C 26, 2247 (1982).

[52] M. Kimura, Phys. Rev. C 69, 044319 (2004).

[53] R. Y. Cusson and H. C. Lee, Nucl. Phys. A211, 429 (1973).

[54] G. K. Tobin, M. C. Ferriss, K. D. Launey, T. Dytrych, J. P. Draayer, A. C. Dreyfuss and C. Bahri, Phys. Rev. C 89 (2014) 034312.

[55] D. R. Tilley, C. M. Cheves, J. H. Kelley, S. Raman, H. R. Weller, Nucl. Phys. A 636, 249 (1998).

[56] P. Raghavan, Atomic Data Nucl. Data Tables 42, 189 (1989).

[57] M. Wang, G. Audi, A. H. Wapstra, F. G. Kondev, M. MacCormick, X. Xu, and B. Pfeiffer, Chin. Phys. C 36,1603 (2012).

[58] D. R. Peterson, and K. T. Hecht, Nucl. Phys. A 344, 361 (1980).

[59] R. B. Firestone, Nucl. Data Sheets 108, 2319 (2007).

[60] T. Bengtsson and I. Ragnarsson, Nucl. Phys. A 436, 14 (1985). 